\title{
The Secondary Organic Aerosol Processor (SOAP v1.0) model: a unified model with different ranges of complexity based on the molecular surrogate approach
}

\author{
F. Couvidat ${ }^{1, *}$ and K. Sartelet ${ }^{1}$ \\ ${ }^{1}$ CEREA, Joint Laboratory École des Ponts ParisTech/EDF R\&D, Université Paris-Est, 77455 Marne-la-Vallée, France \\ * now at: Institut National de l'Environnement Industriel et des Risques, 60550 Verneuil-en-Halatte, France
}

Correspondence to: F. Couvidat (florian.couvidat@ineris.fr)

Received: 6 December 2013 - Published in Geosci. Model Dev. Discuss.: 14 January 2014

Revised: 13 February 2015 - Accepted: 23 March 2015 - Published: 22 April 2015

\begin{abstract}
In this paper the Secondary Organic Aerosol Processor (SOAP v1.0) model is presented. This model determines the partitioning of organic compounds between the gas and particle phases. It is designed to be modular with different user options depending on the computation time and the complexity required by the user. This model is based on the molecular surrogate approach, in which each surrogate compound is associated with a molecular structure to estimate some properties and parameters (hygroscopicity, absorption into the aqueous phase of particles, activity coefficients and phase separation).

Each surrogate can be hydrophilic (condenses only into the aqueous phase of particles), hydrophobic (condenses only into the organic phases of particles) or both (condenses into both the aqueous and the organic phases of particles). Activity coefficients are computed with the UNIFAC (UNIversal Functional group Activity Coefficient; Fredenslund et al., 1975) thermodynamic model for short-range interactions and with the Aerosol Inorganic-Organic Mixtures Functional groups Activity Coefficients (AIOMFAC) parameterization for medium- and long-range interactions between electrolytes and organic compounds. Phase separation is determined by Gibbs energy minimization.

The user can choose between an equilibrium representation and a dynamic representation of organic aerosols (OAs). In the equilibrium representation, compounds in the particle phase are assumed to be at equilibrium with the gas phase. However, recent studies show that the organic aerosol is not at equilibrium with the gas phase because the organic phases could be semi-solid (very viscous liquid phase). The
\end{abstract}

condensation-evaporation of organic compounds could then be limited by the diffusion in the organic phases due to the high viscosity. An implicit dynamic representation of secondary organic aerosols (SOAs) is available in SOAP with OAs divided into layers, the first layer being at the center of the particle (slowly reaches equilibrium) and the final layer being near the interface with the gas phase (quickly reaches equilibrium). Although this dynamic implicit representation is a simplified approach to model condensation-evaporation with a low number of layers and short CPU (central processing unit) time, it shows good agreements with an explicit representation of condensation-evaporation (no significant differences after a few hours of condensation).

\section{Introduction}

Fine particles are regulated because of their impact on human health (WHO, 2003). Furthermore, they degrade atmospheric visibility (Larson et al., 1989) and influence climate change (Kanakidou et al., 2005). It is therefore necessary to develop models able to predict particle formation, which can be used to predict their impact on health and environment and evaluate emission mitigation policies. Particulate organic matter $(\mathrm{OM})$ represents a large fraction of the total fine particulate mass, typically between 20 and $60 \%$ (Kanakidou et al., 2005; Yu et al., 2007; Zhang et al., 2007). Therefore, efforts have to be made to represent OM as accurately as possible in models. Three-dimensional (3-D) air quality models, which estimate particle concentrations, need to have an implicit rep- 
resentation of organic species. Because of the large number of organic species involved originating from diverse anthropogenic and biogenic sources, species need to be lumped according to their properties (e.g., by lumping species with similar saturation vapor pressures). In the surrogate-based methodologies, molecular structures are attached to surrogate compounds representing a large number of secondary organic aerosol (SOA) species to estimate several properties (e.g., condensation into an aqueous phase, oligomerization, hygroscopicity, non-ideality).

In 3-D air quality models, several assumptions are made on the thermodynamics of organic aerosols (OAs) such as equilibrium between the gas phase and the particle phase, ideality or no phase separation. However, these assumptions could strongly impact simulated OA formation. For example, some recent experimental studies emphasize the need to take into account dynamical aspects of the formation of OAs rather than assuming thermodynamic equilibrium with the gas phase because OAs can be highly viscous (Virtanen et al., 2010; Cappa and Wilson, 2011; Pfrang et al., 2011; Shiraiwa et al., 2011; Vaden et al., 2011; Shiraiwa and Seinfeld, 2012; Abramson et al., 2013).

Some OA models already represent the formation and the condensation of organic compounds using the surrogate approach: the Audio Electric Research GmbH (AER)/Electric Power Research Institute (EPRI)/Caltech (AEC) model (Pun et al., 2002, 2006), the Hydrophilic/Hydrophobic Organic model $\left(\mathrm{H}^{2} \mathrm{O}\right)$ (Couvidat et al., 2012, 2013) and the Model to Predict the Multiphase Partitioning of Organics (MPMPO) (Griffin et al., 2003). However, none of these models take into account the dynamics of the condensation of organic compounds, the influence of interactions between organic and inorganic compounds on activity coefficients or the phase separation of particulate OM into several organic phases, whereas the interplay of these phenomena should be taken into account in models (Shiraiwa et al., 2013). Moreover, contrary to MPMPO AEC and $\mathrm{H}^{2} \mathrm{O}$ assume that each compound may condense into only one phase (the organic or the aqueous phase). Computation of activity coefficients and phase separation at equilibrium has been extensively developed in the thermodynamic model Aerosol InorganicOrganic Mixtures Functional groups Activity Coefficients (AIOMFAC) (Zuend et al., 2008, 2010, 2011; Zuend and Seinfeld, 2012). Shiraiwa et al. (2012) developed a multilayer model KM-GAP (kinetic multi-layer model of gasparticle interactions in aerosols and clouds) which explicitly treats the condensation and particle diffusion of organic compounds as well as heat transfer and particle-phase reactions.

To represent organic aerosol formation and take into account non-ideality, phase separation and the viscous state of OAs, the Secondary Organic Aerosol Processor (SOAP), destined to be implemented in 3-D air quality models, is developed and presented here. This model is designed to be modular with different user options depending on the computation time and the complexity required by the user. The user can choose between an equilibrium representation and a dynamic representation of organic aerosols, between ideality and non-ideality (with or without phase separation and interactions with inorganic ions) and on which phases each surrogate compound can condense (the organic phases, the aqueous phase or all phases). This paper describes the SOAP model and the results of several test cases. As the dynamic representation of SOAP is implicit in order to work with low numbers of particle layers and short CPU times, comparisons to an explicit representation of condensation-evaporation are also presented.

\section{Model development}

\subsection{Overview}

SOAP is based on the surrogate approach in which SOA species are divided into three types: hydrophilic species (which condense only into an aqueous phase when an aqueous phase is present, i.e., when the concentration of water in aerosol is higher than $0.01 \mu \mathrm{g} \mathrm{m}^{-3}$ ), hydrophobic species (which condense only into organic phases due to their low affinity with water) or both (which condense into both phases) as chosen by the user. The model can represent the non-ideality of aerosols (interactions between organic compounds and interactions between organic and inorganic compounds represented via activity coefficients), hygroscopicity, phase separation and formation of SOA following an equilibrium approach or a dynamic approach.

Semi-volatile organic compounds (SVOCs) are represented by surrogate compounds. To represent the nonideality of aerosols, molecular structures are assigned by the user to each surrogate compound. A default structure is provided for each surrogate. This structure is used to compute the non-ideality of aerosols via activity coefficients. If the user specifies that a compound is both hydrophilic and hydrophobic, the repartition between phases is done according to the value of activity coefficients. However, the user can decide that a compound is only hydrophilic or only hydrophobic (for example alkane or lowly oxidized compounds are probably not absorbed by the aqueous phase of particles). Moreover, if there is no compound that is both hydrophilic and hydrophobic, the condensation into organic phases can be solved separately from the condensation into the aqueous phase. The system is then uncoupled. On the other hand, if there is at least one compound which is both hydrophilic and hydrophobic, the condensation into organic phases and the condensation into the aqueous phase must be solved simultaneously. The system is then coupled and consumes more CPU time. The user may prefer an uncoupled system for 3-D application due to higher time efficiency.

To compute SOA formation, two different approaches can be used to model gas/particle partitioning: the equilibrium approach and the dynamic approach. In the equilibrium ap- 
proach, aerosols are assumed to be at thermodynamic equilibrium with the gas phase. The model then uses the NewtonRaphson method to efficiently compute the partitioning of each compound between the gas and particle phases. In the dynamic approach, condensation and particle diffusion of organic compounds are treated with a multi-layer representation of OAs (the organic mass is divided into several layers having different characteristic times to reach equilibrium). In this method, the particle size distribution is divided into sections (inside a section/bin, all particles are assumed to have the same diameter). Inside a bin, compounds condense into the aqueous phase or/and the organic phases. Furthermore, each bin can be separated into several layers (the number of layers being the same for each bin) and several phases (the number of phases can change from one bin/layer to another bin/layer).

SOAP is based on the methodology of the $\mathrm{H}^{2} \mathrm{O}$ and AEC model. However, as described above, several processes were added. The model can have species that can condense into the organic phases and the aqueous phase (like in MPMPO). It can compute the effects of the interactions between inorganic and organic compounds on the condensation of organics, phase separation of the organic aerosol, and dynamic formation of SOA. The dynamic approach developed in this study is an implicit approach to take into account particlephase diffusion with a low number of layers and to keep a computation time as low as possible in order to be used by 3-D air quality models. It does not describe particle-phase diffusion as thoroughly as KM-GAP (Shiraiwa et al., 2012).

The code can be downloaded at http://cerea.enpc.fr/en/ modeles.html.

\subsection{Organic aerosol formation at equilibrium}

The fundamental equations used in SOAP to represent the partitioning between gas and particles under the equilibrium assumption are described below.

\subsubsection{Equilibrium between the gas phase and one organic phase}

The equilibrium between the gas phase and one organic phase is described by Raoult's law:

$P_{i}=\gamma_{i, \text { org }} X_{i, \text { org }} P_{i}^{0}$,

where $P_{i}$ is the partial pressure of the compound $i, \gamma_{i, \text { org }}$ the activity coefficient of $i$ in the organic phase, $X_{i, \text { org }}$ the molar fraction of $i$ in the organic phase and $P_{i}^{0}$ the saturation vapor pressure of $i$. Pankow (1994) rewrote Eq. (1) (see Eq. 2) to compute the absorption of organic compounds by an organic phase:

$\frac{A_{\mathrm{p}, i}}{A_{\mathrm{g}, i}}=K_{\mathrm{p}, i} M_{\mathrm{o}}$,

where $A_{\mathrm{p}, i}$ is the concentration of $i$ in the organic phase (in $\mu \mathrm{g} \mathrm{m}^{-3}$ ), $A_{\mathrm{g}, i}$ the concentration of $i$ in the gas phase (in $\mu \mathrm{g} \mathrm{m}^{-3}$ ), $M_{\mathrm{O}}$ the concentration of the organic phase (in $\left.\mu \mathrm{g} \mathrm{m}^{-3}\right)$ and $K_{\mathrm{p}, i}$ the organic-phase partitioning coefficient (in $\mathrm{m}^{3} \mu \mathrm{g}^{-1}$ ) which is computed using Eq. (3) (Pankow, 1994).

$K_{\mathrm{p}, i}=\frac{760 \times 8.202 \times 10^{-5} \times T}{M_{\mathrm{ow}} \gamma_{i, \text { org }} P_{i}^{0} \times 10^{6}}$,

where $T$ is the temperature (in K), $M_{\mathrm{ow}}$ the mean molar mass of the organic phase (in $\mathrm{g} \mathrm{mol}^{-1}$ ) and $P_{i}^{0}$ the saturation vapor pressure (in torr). In SOAP, activity coefficients are computed with the thermodynamic model UNIFAC (UNIversal Functional group Activity Coefficient; Fredenslund et al., 1975). Moreover, $P_{i}^{0}$ or partitioning constants $K_{\mathrm{p}, i}$ are the same as those used in $\mathrm{H}^{2} \mathrm{O}$ (they are generally determined by fitting to experimental results obtained in environmental chambers at a temperature $T_{\text {ref }}$ ). The partitioning coefficient is extrapolated from $T_{\text {ref }}$ to $T$ by using the enthalpy of vaporization $\Delta H_{i}$ (in $\mathrm{J} \mathrm{mol}^{-1}$ ) according to the ClausiusClapeyron equation.

$\frac{K_{\mathrm{p}, i}(T)}{T}=\frac{K_{\mathrm{p}, i}\left(T_{\mathrm{ref}}\right)}{T_{\mathrm{ref}}} \exp \left(\frac{\Delta H_{i}}{R}\left(\frac{1}{T}-\frac{1}{T_{\mathrm{ref}}}\right)\right)$

The absorption of water by the organic phase is computed with Eq. (5) derived from Eq. (1) applied for water.

$A_{\text {p,water }}=\frac{M_{\text {water }} M_{\mathrm{o}} \mathrm{RH}}{\gamma_{\text {water,org }} M_{\mathrm{ow}}}$,

where RH is the relative humidity, $M_{\text {water }}$ the molar mass of water (in $\mathrm{g} \mathrm{mol}^{-1}$ ) and $\gamma_{\text {water,org }}$ the activity coefficient of water in the organic phase.

\subsubsection{Equilibrium between the gas phase and an aqueous phase}

SOAP does not currently take into account the formation of inorganic aerosols. An inorganic aerosol model like ISORROPIA (Nenes et al., 1998) must be called separately and prior to the call of SOAP to provide inputs to SOAP: $\mathrm{pH}$, concentrations of inorganic ions, ionic strength and the liquid water content of aerosols.

The equilibrium between the gas and aqueous phases can be described not only by Raoult's law (Eq. 6) but also by Henry's law (Eq. 7) if infinite dilution is assumed:

$P_{i}=\gamma_{i, \text { aq }} X_{i, \text { aq }} P_{i}^{0}$,

where $\gamma_{i \text {,aq }}$ is the activity coefficient of $i$ in the aqueous phase and $X_{i \text {,aq }}$ the molar fraction of $i$ in the aqueous phase.

$C_{i}=H_{i} P_{i}$,

where $C_{i}$ is the concentration (in $\mathrm{M}$ ) of species $i$ in the aqueous phase, $P_{i}$ in atmosphere and $H_{i}$ the Henry's law constant (in $\mathrm{M} \mathrm{atm}^{-1}$ ). 
As the Henry's law is often used to express the partitioning between the gas and aqueous phases, a modified Henry's law is used to extrapolate infinite dilution conditions to all conditions using an aqueous-phase partitioning coefficient $K_{\mathrm{aq}, i}$ :

$\frac{A_{\mathrm{aq}, i}}{A_{\mathrm{g}, i}}=K_{\mathrm{aq}, i} \mathrm{AQ}$,

where $A_{\mathrm{aq}, i}$ is the concentration of $i$ in the organic phase (in $\mu \mathrm{g} \mathrm{m}^{-3}$ ), $A_{\mathrm{g}, i}$ the concentration of $i$ in the gas phase (in $\mu \mathrm{g} \mathrm{m}^{-3}$ ), AQ the total concentration (organics + inorganics including water) of the aqueous phase (in $\mu \mathrm{g} \mathrm{m}^{-3}$ ) and $K_{\text {aq }, i}$ the aqueous-phase partitioning coefficient (in $\mathrm{m}^{3} \mu \mathrm{g}^{-1}$ ) which is computed with Eq. (9):

$K_{\mathrm{aq}, i}=\frac{H_{i} \mathrm{RT}}{\rho_{\text {water }} \zeta_{i} \times 1.013 \times 10^{11}} \times \frac{18}{M_{\mathrm{aq}}}$,

where $\rho_{\text {water }}$ is the density of the aqueous phase (in $\mathrm{kg} \mathrm{m}^{-3}$ ), $M_{\mathrm{aq}}$ the molar mass of the aqueous phase (in $\mathrm{g} \mathrm{mol}^{-1}$ ), which can be slightly different from the molar mass of water due to the presence of other compounds, and $\zeta_{i}$ the activity coefficient by reference to infinite dilution. $\zeta_{i}$ is computed with Eq. (10):

$\zeta_{i}=\frac{\gamma_{i, \mathrm{aq}}}{\gamma_{i, \mathrm{aq}}^{\infty}}$

where $\gamma_{i, \text { aq }}^{\infty}$ is the activity coefficient at infinite dilution in water, which is computed with UNIFAC. However, the original UNIFAC (Fredenslund et al., 1975) only computes the activity coefficients due to short-range interactions and does not take into account medium- and long-range interactions due to the presence of electrolytes in the aqueous phase. In the aqueous phase, activity coefficients are computed from Eq. (11) (Zuend et al., 2008)

$\gamma_{i, \text { aq }}=\gamma_{\mathrm{LR}} \gamma_{\mathrm{MR}} \gamma_{\mathrm{SR}}$

$\gamma_{\mathrm{LR}}, \gamma_{\mathrm{MR}}$ and $\gamma_{\mathrm{SR}}$ are respectively the activity coefficients at long-, medium- and short-range interactions. $\gamma_{\mathrm{SR}}$ is computed with UNIFAC, whereas $\gamma_{\text {LR }}$ and $\gamma_{\text {MR }}$ are computed with the AIOMFAC method. The last two parameters model the influence of inorganic ions on the partitioning of organic compounds (Zuend et al., 2008, 2011; Zuend and Seinfeld, 2012).

Similarly to the case of condensation into an organic phase (Eq. 4), the partitioning coefficient is extrapolated from $T_{\text {ref }}$

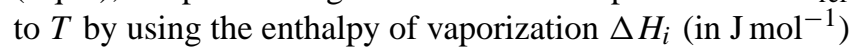
as described in Eq. (12):

$\frac{K_{\mathrm{aq}, i}(T)}{T}=\frac{K_{\mathrm{aq}, i}\left(T_{\mathrm{ref}}\right)}{T_{\mathrm{ref}}} \exp \left(\frac{\Delta H_{i}}{R}\left(\frac{1}{T}-\frac{1}{T_{\mathrm{ref}}}\right)\right)$.

However, some compounds are acids that can dissociate in the aqueous phase. Therefore, partitioning coefficients are modified to take into account acidic dissociation as done by Pun et al. (2006).
The absorption of water by the aqueous phase is computed with Eq. (13) derived from Eq. (1)

$A_{\text {aq, water }}=\frac{M_{\text {water }} \mathrm{AQ} \times \mathrm{RH}}{\gamma_{\text {water,aq }} M_{\text {aq }}}$

where $\gamma_{\text {water,aq }}$ is the activity coefficient of water in the aqueous phase. As the amount of water absorbed by inorganics is already given by the inorganic model (i.e., ISORROPIA) and is used as input for SOAP, we assumed that the total amount of water (from inorganics and organics) computed by SOAP should at least be equal to the amount of water given by the inorganic model. Therefore, if the amount of water computed by SOAP is lower than the amount computed by the inorganic model, it is replaced by the amount computed by the inorganic model. This problem arises because inorganics are not computed within SOAP (in that case, there would be no numerical issue) and water concentrations can be lower in SOAP than in the inorganic model ISORROPIA for several reasons. First, there can be numerical differences between SOAP and ISORROPIA because different parameterizations are used in SOAP and ISORROPIA to compute water concentrations: SOAP computes the amount of water using the water activity computed by the thermodynamic models UNIFAC and AIOMFAC, whereas ISORROPIA computes the amount of water using the Zdanovskii-Stokes-Robinson (ZSR) correlation (Robinson and Stokes, 1965). Second, the amount of water absorbed by the aerosol can be different from the sum of the water amount absorbed by inorganics and the water amount absorbed by organics. Choi and Chan (2002) found that organic species can either reduce or enhance the water absorption of inorganic compounds. Depending on the conditions, the amount of water computed by SOAP could be higher without organics than with. Here we chose to assume that the amount of water should at least be equal to the amount of water given by ISORROPIA, although it would be possible to keep the amount of water computed by SOAP even when lower than the amount of water computed by ISORROPIA. However, if the amount of water given by SOAP is significantly lower than ISORROPIA, this would induce changes in the amount of inorganics in the aerosol. As the amount of inorganics may not be recomputed after SOAP, we considered that the amount of water is at least equal to the amount of water given by ISORROPIA; that is, the amount of water being recomputed only to provide a better estimate of the amount of water when water absorption are mainly due to organics. The best way to deal with this dilemma would be to fully couple inorganic and organic aerosol formation.

\subsubsection{Equilibrium between the gas phase and several particulate phases}

SOAP can compute the partitioning of compounds between the gas phase and several particulate phases. The user can specify for each compound if it is hydrophilic (condense into 
the aqueous phase of particles) or hydrophobic (condense into the organic phase of particles) or both hydrophilic and hydrophobic (condense into both phases).

For an uncoupled system (compounds cannot be both hydrophobic and hydrophilic), for hydrophobic compounds, the total concentration of $i$ in all phases $A_{\text {tot }, i}$ is

$A_{\mathrm{tot}, i}=A_{\mathrm{p}, i}+A_{\mathrm{g}, i}$

which when combined with Eq. (2) results in

$A_{\mathrm{p}, i}=A_{\mathrm{tot}, i} \frac{K_{\mathrm{p}, i} M_{\mathrm{o}}}{1+K_{\mathrm{p}, i} M_{\mathrm{o}}}$.

A method of Newton-Raphson is then used to solve Eq. (15) and to minimize the error (an accuracy threshold is provided by the user):

error $=M_{\mathrm{o}}-\sum_{i} A_{\mathrm{tot}, i} \frac{K_{\mathrm{p}, i} M_{\mathrm{o}}}{1+K_{\mathrm{p}, i} M_{\mathrm{o}}}$.

For an uncoupled system, for hydrophilic compounds, the total concentration of $i$ in all phases $A_{\mathrm{tot}, i}$ is

$A_{\mathrm{tot}, i}=A_{\mathrm{aq}, i}+A_{\mathrm{g}, i}$

which when combined with Eq. (8) results in

$A_{\text {aq }, i}=A_{\text {tot }, i} \frac{K_{\text {aq }, i} \mathrm{AQ}}{1+K_{\text {aq }, i} \mathrm{AQ}}$.

A method of Newton-Raphson is then used to solve Eq. (18) and to minimize the error (an accuracy threshold is provided by the user):

error $=\mathrm{AQ}-\mathrm{AQ}_{\text {inorg }}-\sum_{i} A_{\text {tot }, i} \frac{K_{\mathrm{aq}, i} \mathrm{AQ}}{1+K_{\mathrm{aq}, i} \mathrm{AQ}}$,

where $\mathrm{AQ}_{\text {inorg }}$ is the concentration of inorganic compounds in the aqueous phase. For a coupled system, the total concentration of $i$ in all phases $A_{\mathrm{tot}, i}$ is

$A_{\mathrm{tot}, i}=A_{\mathrm{p}, i}+A_{\mathrm{aq}, i}+A_{\mathrm{g}, i}$

which when combined with Eqs. (2) and (8) results in

$$
\begin{aligned}
A_{\mathrm{p}, i} & =A_{\mathrm{tot}, i} \frac{K_{\mathrm{p}, i} M_{\mathrm{o}}}{1+K_{\mathrm{aq}, i} \mathrm{AQ}+K_{\mathrm{p}, i} M_{\mathrm{o}}}, \\
A_{\mathrm{aq}, i} & =A_{\mathrm{tot}, i} \frac{K_{\mathrm{aq}, i} \mathrm{AQ}}{1+K_{\mathrm{aq}, i} \mathrm{AQ}+K_{\mathrm{p}, i} M_{\mathrm{o}}} .
\end{aligned}
$$

Similarly, a method of Newton-Raphson is used to simultaneously solve Eqs. (21) and (22) and to minimize the errors $\left(\operatorname{error}_{\mathrm{p}}\right.$ and error $\left._{\mathrm{aq}}\right)$.

$$
\begin{aligned}
\text { error }_{\mathrm{p}}= & M_{\mathrm{o}}-\sum_{i} A_{\mathrm{tot}, i} \frac{K_{\mathrm{p}, i} M_{\mathrm{o}}}{1+K_{\mathrm{aq}, i} \mathrm{AQ}+K_{\mathrm{p}, i} M_{\mathrm{o}}} \\
\text { error }_{\mathrm{aq}}= & \mathrm{AQ}-\mathrm{AQ}_{\mathrm{inorg}}- \\
& \sum_{i} A_{\mathrm{tot}, i} \frac{K_{\mathrm{aq}, i} \mathrm{AQ}}{1+K_{\mathrm{aq}, i} \mathrm{AQ}+K_{\mathrm{p}, i} M_{\mathrm{o}}}
\end{aligned}
$$

\subsubsection{Saturation and separation of organic phases}

If compounds having a low affinity with each other coexist inside a single organic phase, the organic phase may become saturated by some compounds and may become unstable. In this case, the separation of the organic phase into several organic phases (having different composition) may occur. To determine whether separating the organic phase into several organic phases makes the system more stable, the Gibbs energy $G$ is computed as in Erdakos and Pankow (2004), Zuend et al. (2010), and Zuend and Seinfeld (2012) using different system configurations (different number of phases):

$G=\sum_{i} \sum_{\phi} n_{i}^{\phi} \mu_{i}^{\phi}$,

where $\phi$ is an index of the various phases (gaseous and liquid), $n_{i}^{\phi}$ the number of moles of species $i$ in phase $\phi$ and $\mu_{i}^{\phi}$ the chemical potential of $i$ in phase $\phi$.

The most stable configuration of the system has the lowest Gibbs energy. Therefore, by adding one organic phase the Gibbs energy decreases, the system is more stable and phase separation takes place. If the Gibbs energy does not decrease, the previous solution (before adding one organic phase) is more stable and is kept. The number of organic phases is determined iteratively: one phase is added until the Gibbs energy increases.

The partitioning of organic compounds into an aerosol with several organic phases are determined with Eqs. 21 and 22 generalized to several organic phases:

$$
\begin{aligned}
A_{\mathrm{p}, i}^{\mathrm{phase}} & =A_{\mathrm{tot}, i} \frac{K_{\mathrm{p}, i}^{\mathrm{phase}} M_{\mathrm{o}}^{\mathrm{phase}}}{1+K_{\mathrm{aq}, i} \mathrm{AQ}+\sum_{\phi} K_{\mathrm{p}, i}^{\phi} M_{\mathrm{o}}^{\phi}}, \\
A_{\mathrm{aq}, i} & =A_{\mathrm{tot}, i} \frac{K_{\mathrm{aq}, i} \mathrm{AQ}}{1+K_{\mathrm{aq}, i} \mathrm{AQ}+\sum_{\phi} K_{\mathrm{p}, i}^{\phi} M_{\mathrm{o}}^{\phi}} .
\end{aligned}
$$

\subsection{Dynamic gas uptake by organic particles}

The dynamic approach, which is presented hereafter, is an implicit representation to take into account particle-phase diffusion with a low number of layers, and have a computation time as low as possible to be used in 3-D air quality models. The main assumptions are described here.

The first assumption is that the organic-phase diffusion coefficient is constant over the entire particle. It does not depend on the distance to the center of the particle. Although this assumption may not be valid, it is reasonable because, currently, to our knowledge, there is no parameterization to evaluate the order of magnitude of organic-phase diffusion coefficients.

The second assumption is that in the model there is no direct exchange of compounds between layers, and that the compounds condense directly from the gas phase to the layer or that they evaporate directly from the layer to the gas phase by taking into account an equilibration time specific of the 
layer. Compounds condense into a layer or evaporate from a layer as if the other layers had the same affinity with compounds. Effects of entrapment of compounds inside central layers by the layers closer to the gas-phase interface (compounds inside the central layers having a low affinity with the compounds of the layers at the interface will not be able to evaporate, whereas compounds having a high affinity with the central layer but having a low affinity with the layers at the interface will not be able to condense into the central layer) are not taken into account. The model should give, however, a good estimation of the capacity of the organic aerosol to absorb compounds (by taking into account the time for each layer to reach equilibrium due to diffusion in the particle).

The third assumption is that the organic phases and the aqueous phase evolve separately, i.e., there is no kinetic transfer of compounds between the organic phases and the aqueous phase, due to the complexity of representing properly those transfers, which should strongly depend on the morphology of particles. If a compound tends to go from the aqueous phase to the organic phases, it has first to go from the aqueous phase to the gas phase and then from the gas phase to the organic phases. For example, in case of evaporation of the aqueous phase (that can be due to a strong change of the RH), this assumption can create some evaporation/recondensation issue in the model (compounds evaporate and recondense after some time into the organic phases according to condensation-evaporation fluxes). Actually, a part of organic compounds should go directly from the aqueous phase to the organic phases. It could also be possible that if an aqueous phase coexists with an organic phase into the same particle, an organic compounds will not condense directly from the gas phase into the organic phases (because the kinetic is too slow) but condense first into the aqueous phase and then go from the aqueous phase to the organic phases (if it is quicker for a compound to condense into the organic phases by this pathway). However, it can also be argued that if there is an aqueous phase (the RH is high), the organic phases may not be significantly viscous and therefore a high organic-phase diffusion coefficient should be used.

Finally, the model assumes that there is no gradient of the gas-phase concentrations near the interface with the particle. If the particle is divided into two separated regions (one aqueous and one organic), an angular gradient of gas-phase concentrations could influence the condensation of compounds into the two regions. To address properly this phenomenon, the particle and the gas phase at the vicinity of the particle should be discretized as a function of the angular gradient. However, due to the high diffusivity of organic compounds in the gas phase, the diffusion of organic compounds around the particle should be very quickly compared to the kinetic of condensation and therefore this assumption should have a low effect on condensation-evaporation.

\subsubsection{Diffusion of organic compounds in spherical organic particles}

The diffusion of organic compounds at a radius $r$ at time $t$ inside a spherical particle is governed by the following equation (Seinfeld and Pandis, 1998)

$$
\frac{\partial C}{\partial t}=D_{\mathrm{org}}\left(\frac{\partial^{2} C}{\partial r^{2}}+\frac{2}{r} \frac{\partial C}{\partial r}\right)+R_{\mathrm{org}}(r, t),
$$

where $C(r, t)$ is the molar concentration at radius $r$ at time $t, D_{\text {org }}$ the organic-phase diffusivity and $R_{\text {org }}$ the organicphase reaction rate. By assuming $D_{\text {org }}$ constant, the solution of this equation (with $C(r, 0)=0$ and $C\left(R_{\mathrm{p}}, t\right)=C_{\mathrm{s}}, R_{\mathrm{p}}$ being the radius of the particle) without organic-phase reaction is according to Seinfeld and Pandis (1998):

$$
\frac{C(r, t)}{C_{\mathrm{s}}}=1+\frac{R_{\mathrm{p}}}{r} \sum_{n=1}^{\infty}(-1)^{n} \frac{2}{n \pi} \sin \frac{n \pi r}{R_{\mathrm{p}}} \exp \left(-\frac{n^{2} \pi^{2} D_{\mathrm{org}} t}{R_{\mathrm{p}}^{2}}\right) .
$$

By integrating Eq. (29) over the volume of a spherical particle, the following equation is found for the concentration in the particle phase $A_{\mathrm{p}}$ :

$$
\frac{A_{\mathrm{p}}(t)}{A_{\mathrm{eq}}}=1-\sum_{n=1}^{\infty} \frac{6}{n^{2} \pi^{2}} \exp \left(-\frac{n^{2} t}{\tau_{\mathrm{dif}}}\right),
$$

where $\tau_{\text {dif }}$ is the characteristic time for diffusion in the center of the particle:

$\tau_{\mathrm{dif}}=\frac{R_{\mathrm{p}}^{2}}{\pi^{2} D_{\mathrm{org}}}$,

where $A_{\mathrm{eq}}$ is the organic-phase concentration at equilibrium with the gas phase $\left(A_{\mathrm{eq}}=K_{\mathrm{p}} M_{\mathrm{o}} A_{\mathrm{g}}\right)$. In Eq. (30), $A_{\mathrm{p}}$ can be interpreted as the sum of an infinity number of layers of concentration $A_{\mathrm{p}}^{\text {layer }}$ :

$$
\begin{aligned}
A_{\mathrm{p}} & =\sum_{\text {layer }=1}^{\infty} A_{\mathrm{p}}^{\text {layer }}, \\
A_{\mathrm{p}}^{\text {layer }} & =V_{\text {layer }} A_{\text {eq }}\left(1-\exp \left(-\frac{\alpha_{\text {layer }} t}{\tau_{\text {dif }}}\right)\right),
\end{aligned}
$$

where $A_{\mathrm{p}}^{\text {layer }}$ is the concentration (in $\mu \mathrm{g} \mathrm{m}^{-3}$ ) in a layer of volume fraction $V_{\text {layer }}$ determined by the fraction of the volume of the particle constituted by the layer and $\alpha_{\text {layer }}$ the ratio between the characteristic diffusion time at the center of the particle $\tau_{\text {dif }}$ to the characteristic diffusion time $\tau_{\text {dif }}^{\text {layer }}$ of the layer such as

$\alpha_{\text {layer }}=\tau_{\mathrm{dif}} / \tau_{\mathrm{dif}}^{\text {layer }}$.

Numerically, Eq. (30) can be approached by a finite number of diffusion layers $N_{\text {layer }}^{\text {dif }}$ (number of layers into which 


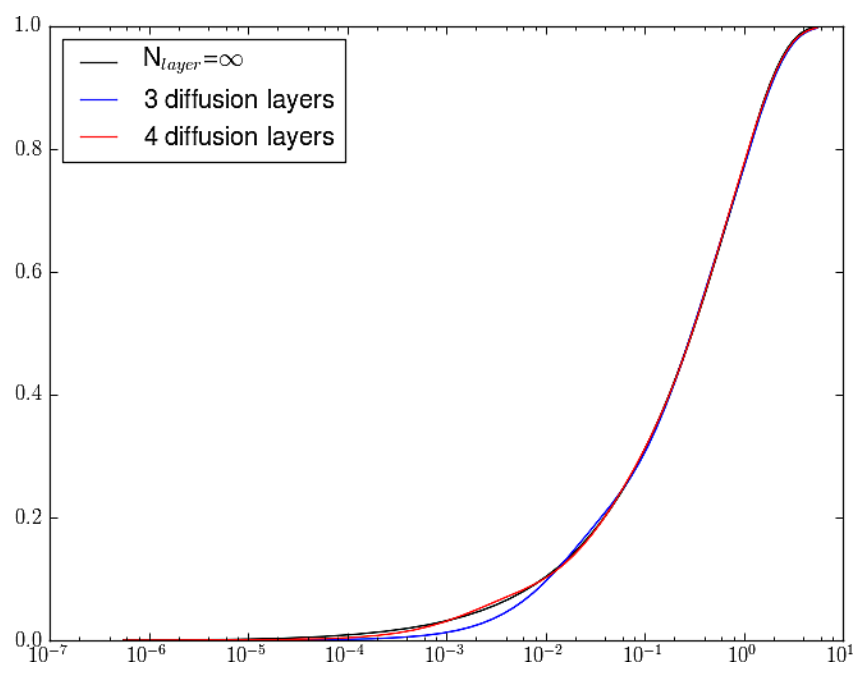

Figure 1. Evolution of the ratio $A_{\mathrm{p}}(t) / A_{\mathrm{eq}}$ as a function of the ratio $t / \tau_{\text {dif }}$.

diffusion occurs) and by fitting the parameters $V_{\text {layer }}$ and $\alpha_{\text {layer }}$ such as

$A_{\mathrm{p}}=\sum_{\text {layer }=1}^{N_{\text {layer }}^{\text {dif }}} A_{\mathrm{p}}^{\text {layer }}$.

First, for a given concentration $A_{\text {eq }}$, a given $D_{\text {org }}$ or $R_{\mathrm{p}}$, we estimate $A_{\mathrm{p}}(t)$ using Eq. (32). Then $V_{1}, V_{2}, V_{3}, \alpha_{1}, \alpha_{2}$, $\alpha_{3}$ are fitted such as satisfying Eq. (35). The values of these parameters do not depend of the choice of $A_{\mathrm{eq}}, D_{\mathrm{org}}$ and $R_{\mathrm{p}}$.

For example, if we choose to have three diffusion layers, then $V_{1}=0.6, V_{2}=0.26, V_{3}=0.14, \alpha_{1}=0.9878$, $\alpha_{2}=6.2558$ and $\alpha_{3}=68.8666$. For four diffusion layers, we use $V_{1}=0.608, V_{2}=0.218, V_{3}=0.121, V_{4}=0.053$, $\alpha_{1}=0.998, \alpha_{2}=4.904, \alpha_{3}=30.98$ and $\alpha_{4}=663.4$. Equation (35) gives a good approximation of Eq. (32) as shown in Fig. 1 with three and four diffusion layers, although with three diffusion layers diffusion is underestimated for low concentrations (lower than one tenth of the concentration at equilibrium).

The evolution of concentrations $A_{\mathrm{p}, i}^{\text {bin,layer }}$ in a bin and a layer due to the condensation of species $i$ limited by the diffusion of organic compounds in the organic phase is described by the Eq. (36), which describes the flux of diffusion $J_{\text {diff }, i}^{\text {bin,layer }}$, i.e., the evolution of the concentration $A_{\mathrm{p}, i}^{\text {bin,layer }}$ compared to an equilibrium concentration (Pankow model: $A_{\mathrm{eq}}^{\text {bin,layer }}=A_{\mathrm{g}, i} K_{\mathrm{p}, i}^{\mathrm{bin}, \text { layer }} M_{\mathrm{o}}^{\text {bin,layer }}$ ) (with the assumption that the concentrations in one layer can be described independently from the concentrations in the other layers).

$$
\begin{aligned}
& J_{\text {diff }, i}^{\text {bin,layer }}=\frac{\mathrm{d} A_{\mathrm{p}, i}^{\text {bin,layer }}}{\mathrm{d} t}= \\
& k_{\text {diffusion }}\left(A_{\mathrm{g}, i} K_{\mathrm{p}, i}^{\text {bin,layer }} M_{\mathrm{o}}^{\text {bin,layer }}-A_{\mathrm{p}, i}^{\text {bin,layer }}\right),
\end{aligned}
$$

where $M_{\mathrm{o}}^{\text {bin,layer }}$ is the total mass of the organic phase in the layer computed with Eq. (37) by assuming that the density of the OM is constant over layers and $k_{\text {diffusion }}$ the kinetic coefficient for diffusion (in s${ }^{-1}$ ) computed with Eq. (38) as the inverse for the characteristic diffusion time in a layer.

$$
\begin{aligned}
M_{\mathrm{o}}^{\text {bin,layer }} & =V_{\text {layer }} M_{\mathrm{o}}^{\text {bin }} \\
k_{\text {diffusion }} & =\frac{\alpha_{\text {layer }}}{\tau_{\text {dif }}}
\end{aligned}
$$

\subsubsection{Diffusion of organic compounds in more complex particles}

The previous equations correspond to the diffusion of organic compounds into an entirely organic spherical particle. However, in the atmosphere, particles generally have more complex geometries and can also be constituted by solid and/or inorganic phases. The morphology affects the time for an organic phase to be diffused in the particle. For example, for a same particle diameter, a particle entirely organic needs more time to reach equilibrium with the gas phase than a particle constituted by a solid core in the center surrounded by an organic phase (because the organic compounds do not penetrate the particle all the way to the center, they are only diffused inside the coating). As diffusion of organic compounds is affected by the presence of solid phases, morphology factors are designed to take into account this solid phase where diffusion of organics may not occur. We assume that this solid phase is located at the core of particles based on Katrinak et al. (1993), Sachdeva and Attri (2007), and Wang et al. (2014). Models, such as KM-GAP, treating explicitly the diffusion of compounds inside particles do not need those factors. As in SOAP, the volume of layer has to be constant, whereas only the characteristic time for diffusion can be affected by the morphology. We propose here to parameterize the effects of a solid core by defining morphology factors $f_{\text {morph }}^{\text {layer }}$, which affect the time to reach equilibrium with the gas phase. The characteristic time for diffusion of a layer $\tau_{\text {dif }}^{\text {layer }}$ expressed in Eq. (34) becomes

$$
\tau_{\text {dif }}^{\text {layer }}=\frac{\tau_{\text {dif }}}{f_{\text {morph }}^{\text {layer }} \alpha_{\text {layer }}} .
$$

Equation (36) then becomes

$$
\begin{aligned}
& J_{\text {diff, } i}^{\text {bin,layer }}=\frac{\mathrm{d} A_{\mathrm{p}, i}^{\text {bin,layer }}}{\mathrm{d} t}= \\
& \quad f_{\text {morph }}^{\text {layer }} k_{\text {diffusion }}\left(A_{\mathrm{g}, i} K_{\mathrm{p}, i}^{\text {bin,layer }} M_{\mathrm{o}}^{\text {bin,layer }}-A_{\mathrm{p}, i}^{\text {bin,layer }}\right)
\end{aligned}
$$

with $k_{\text {diffusion }}$ defined as in Eq. (38).

The morphology factors can be determined numerically (at least for some simple case). We determined here morphology factors in the case of a spherical particle with a solid core at 

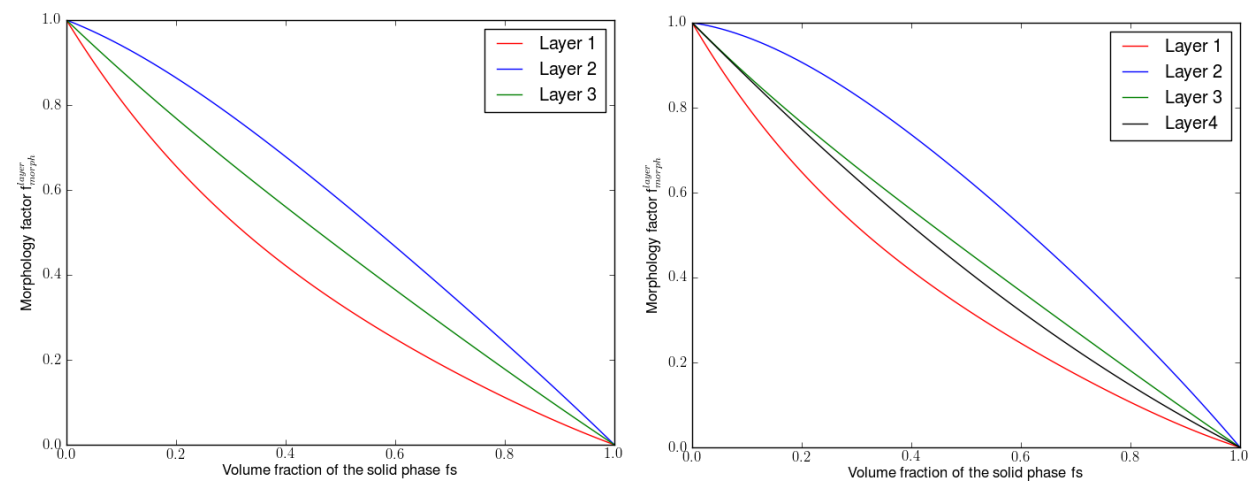

Figure 2. Morphology factors as a function of the volume fraction of the solid phase (layer 1 corresponds to the layer near the solid core of the particle and layer 3 to the layer near the interface) for three diffusion layers (left) and four diffusion layers (right).

the center of the particle. The differential equation for diffusion (Eq. 28) is solved and morphology factors are fitted to minimize the differences between Eqs. (28) and (40) for various volume fractions of the solid phase $f_{\mathrm{s}}$ (volume of the solid phase in the particle over the volume of the particle). This fitting procedure is described in more detail in the Supplement. The variations of the morphology factors with the volume fraction $f_{\mathrm{s}}$ are shown in Fig. 2 for three and four diffusion layers. The morphology factors for a solid core particle can be represented by a polynomial expression such as

$$
\begin{aligned}
f_{\text {morph }}^{\text {layer }}= & A_{\text {layer }} f_{\mathrm{s}}^{4}+B_{\text {layer }} f_{\mathrm{s}}^{3} \\
& +C_{\text {layer }} f_{\mathrm{s}}^{2}+D_{\text {layer }} f_{\mathrm{s}}+1 .
\end{aligned}
$$

The values of the polynomial parameters are shown in Table 2. Typically, the presence of a solid phase would result in a morphology factor greater than 1 , reducing the characteristic diffusion time of organics in the particle. This parameterization only takes into account the simple case of a spherical solid core at the center and not the wide range of morphologies present in the atmosphere. A similar methodology or a methodology based on observations could be applied to other morphologies which could greatly affect the characteristic times for diffusion. For example, morphology factors for the effect of the presence of an aqueous phase on characteristic time for diffusion could be determined (at least as a function of the mass of the aqueous phase and the affinity of compounds with water). Currently, the model assumes there is no effect of the aqueous phase on the characteristic times.

\subsubsection{Condensation-evaporation of organic compounds into a viscous particle}

In this part, the methodology to compute condensationevaporation-diffusion inside a viscous particle is described. The algorithm to compute condensation-evaporationdiffusion fluxes is divided into two steps shown in Figs. 3 and 4. In the first step, the total flux of condensationevaporation-diffusion over the whole particle is computed whereas in the second step this flux is redistributed among layers. The complete methodology to compute fluxes is described hereafter.

The evolution equation of concentration of species $i$ by diffusion in each bin and layer is described by Eq. (40). To properly take into account the condensation-evaporation of low volatile compounds, a thin surface layer, which is not limited by diffusion, is added to the model. In case of very low diffusivity, this layer allows non-volatile and low volatility compounds to condense at the surface of particles without any limitation due to diffusion. Let us denote the number of layers $N_{\text {layer }}$ into which the dynamic of condensationevaporation-diffusion is solved. We have

$N_{\text {layer }}=N_{\text {layer }}^{\text {diff }}+1$.

A volume fraction $V_{\text {layer }}$ for the interface layer of 0.01 is used (using a lower volume fraction does not significantly change the results). Adding an interface layer between the gas and the particle slightly reduces the volume of the other layers, as described in Sect. 2.3.1. In this paper, simulations are performed with four layers (three diffusion layers: $V_{1}=$ $0.6, V_{2}=0.26, V_{3}=0.13$ and $V_{4}=0.01$ ) and with five layers (four diffusion layers: $V_{1}=0.608, V_{2}=0.218, V_{3}=0.121$, $V_{4}=0.043$ and $V_{5}=0.01$ ).

The flux of diffusion in the upper layer (the interface) is zero, as diffusion should not limit absorption at the interface:

$J_{\text {diff }}^{\text {bin,interface }}=0$.

The evolution of concentrations by condensationevaporation at the interface, i.e., the flux of condensationevaporation at the interface, is

$J_{i}^{\text {bin,interface }}=k_{\mathrm{absorption}}\left(A_{\mathrm{g}, i}-A_{\mathrm{g}, i}^{\mathrm{eq}, \mathrm{bin}, \text { interface }}\right)$,

where $A_{\mathrm{g}, i}^{\text {eq,bin,interface }}$ is the gas-phase concentration at equilibrium with the interface layer and $k_{\text {absorption }}$ the kinetic rate 
Table 1. Algorithm to compute the partitioning of compounds at equilibrium in the dynamic approach.
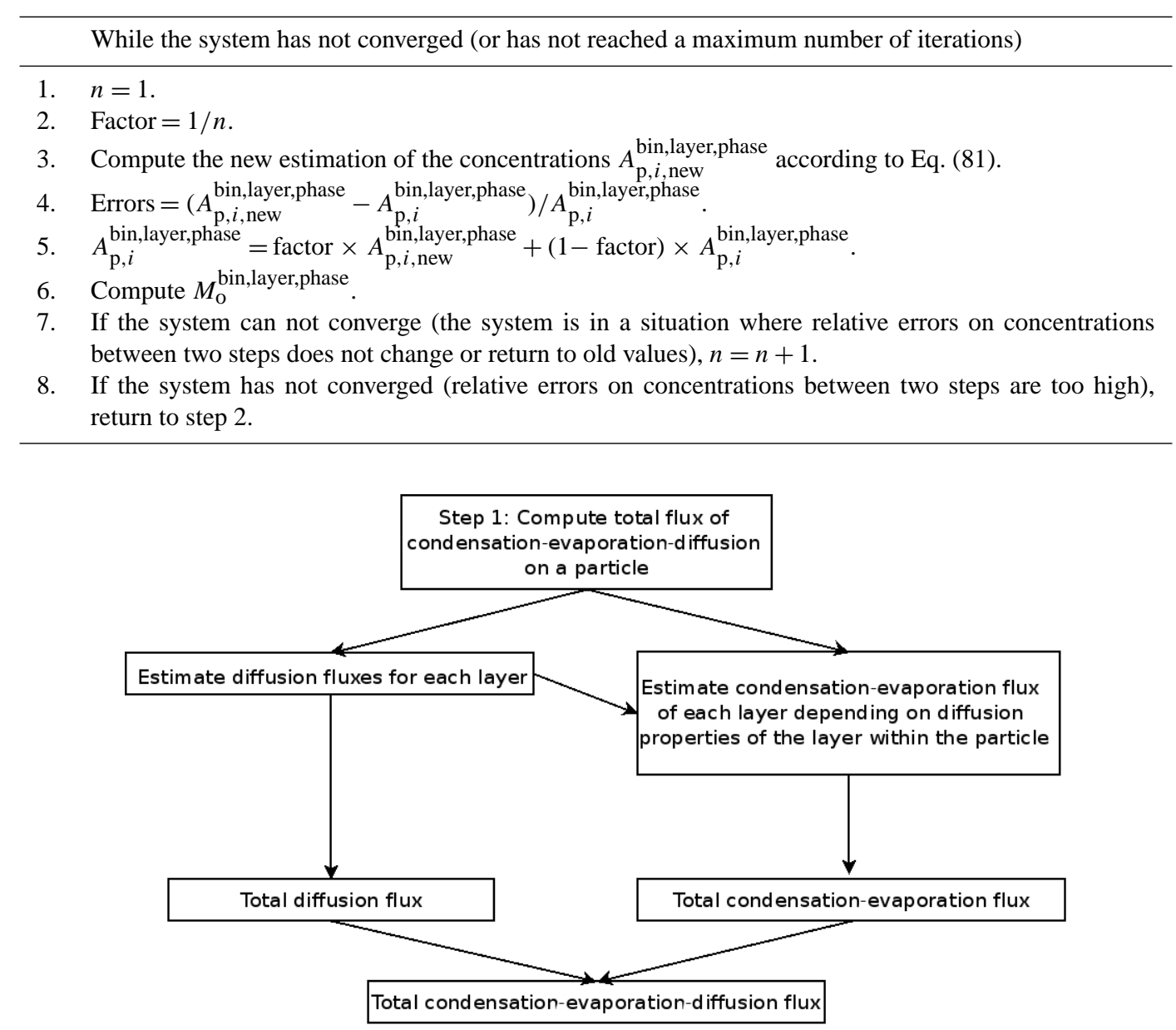

Figure 3. Diagram of the first step of the algorithm to compute evaporation-condensation-diffusion fluxes.

of absorption, which is (Seinfeld and Pandis, 1998)

$k_{\text {absorption }}=2 \pi D_{\mathrm{p}} D_{\text {air }} N^{\text {bin }} f(K n, \alpha)$,

where $D_{\mathrm{p}}$ is the diameter of the particle, $D_{\text {air }}$ the diffusivity of compound $i$ in air and $f(K n, \alpha)$ the transition regime formula of Fuchs and Sutugin (1971).

The gas-phase concentration at equilibrium with the interface layer $A_{\mathrm{g}, i}^{\mathrm{eq}, i}$,interface is computed as follows:

$A_{\mathrm{g}, i}^{\mathrm{eq}, \text { bin,interface }}=K_{\mathrm{effect}} \frac{A_{\mathrm{p}, i}^{\mathrm{bin}, \text { interface }}}{K_{\mathrm{p}, i}^{\mathrm{bin}, \text { interface }} M_{\mathrm{O}}^{\text {bin,interface }}}$,

where $K_{\mathrm{p}, i}^{\mathrm{bin}, \text { interface }}$ is the partitioning coefficient into the interface layer for compound $i$ and $M_{\mathrm{o}}^{\text {bin,interface }}$ the total organic mass concentrations of the interface layer and $K_{\text {effect }}$ the Kelvin effect correction coefficient:

$K_{\text {effect }}=\exp \left(-\frac{2 \sigma M_{\mathrm{ow}}^{\mathrm{bin}}}{\mathrm{RT} \rho_{\text {organic }}}\right)$, where $\sigma$ is the surface tension, $\rho_{\text {organic }}$ the density of the organic phase, $M_{\mathrm{ow}}$ the mean molar mass of the organic phase at the surface of the particle (molar mass of the layer at the interface) and $R_{\mathrm{p}}$ the radius of the particle. A surface tension of $24 \mathrm{mN} \mathrm{m}^{-1}$ is chosen, which is roughly the surface tension of organic compounds according to Seinfeld and Pandis (1998) and a density of the organic phase of $1300 \mathrm{~kg} \mathrm{~m}^{-3}$ is used.

In a comprehensive dynamic model, diffusion would be represented explicitly. However, to have a model with a low number of layers and gain CPU time, the evolution of the concentrations in each layer is solved by considering the combined effect of condensation-evaporation and diffusion in the particle. The flux of condensation-evaporationdiffusion of a compound $i$ inside a bin and a layer is noted $J_{\text {tot, } i}^{\text {bin,layer }}$ and is used to compute the evolution of concentrations of a compound $i$ inside a layer:

$\frac{\mathrm{d} A_{\mathrm{p}, i}^{\text {bin,layer }}}{\mathrm{d} t}=J_{\text {tot }, i}^{\text {bin,layer }}$. 


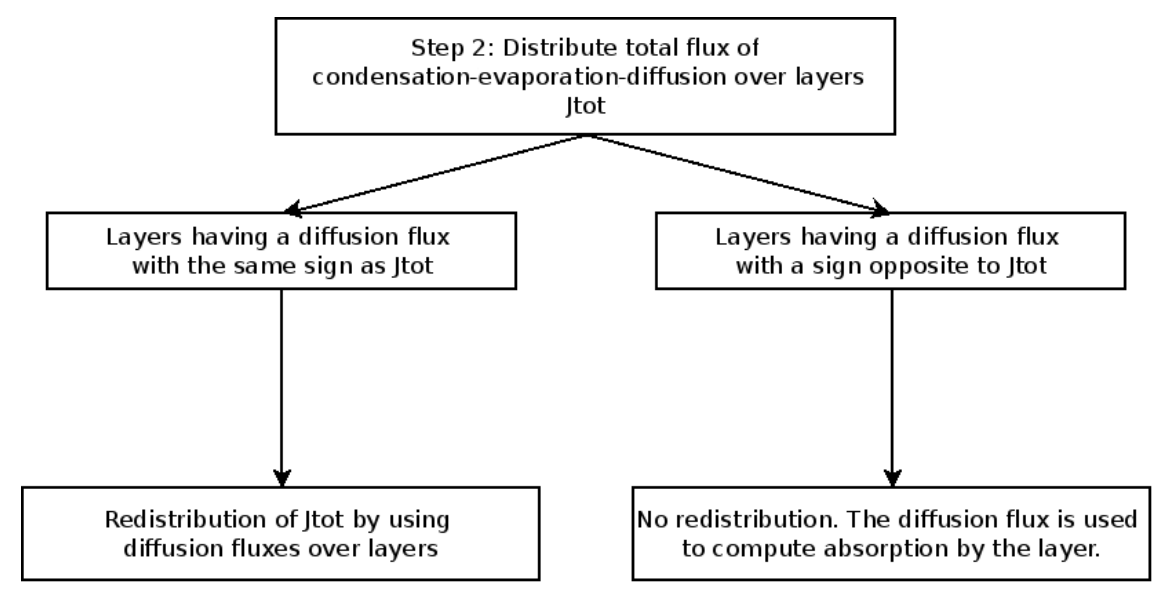

Figure 4. Diagram of the second step of the algorithm to compute evaporation-condensation-diffusion fluxes.

The evolution of the total mass of the particle $J_{\text {tot }, i}^{\text {bin }}$ can be computed by assuming that the characteristic time of the combined effect of condensation-evaporation and diffusion is equal to the sum of the characteristic times of condensation-evaporation and diffusion:

$\frac{1}{J_{\mathrm{tot}, i}^{\text {bin }}}=\frac{1}{J_{\mathrm{cond} / \mathrm{evap}, i}^{\text {bin }}}+\frac{1}{J_{\mathrm{diff}, i}^{\mathrm{bin}}}$,

where $J_{\text {cond/evap, } i}^{\text {bin }}$ is the flux limited by condensationevaporation and $J_{\text {diff }, i}^{\text {bin }}$ the flux limited by diffusion. $J_{\text {diff }, i}^{\text {bi }}$ is the sum of the diffusion fluxes over all bins (each of them being computed by Eq. 40):

$J_{\text {diff }, i}^{\text {bin }}=\sum_{\text {layer }}^{N_{\text {layer }}} J_{\text {diff }, i}^{\text {bin,layer }}$.

$J_{\text {cond/evap }, i}^{\text {bin }}$ is obtained similarly to the interface flux of condensation-evaporation

$J_{\text {cond/evap }, i}^{\text {bin }}=k_{\text {absorption }}\left(A_{\mathrm{g}, i}-A_{\mathrm{g}, i}^{\mathrm{eq}, \mathrm{bin}}\right)$,

where $A_{\mathrm{g}, i}^{\mathrm{eq}, i \mathrm{in}}$ is the gas-phase concentration at equilibrium with the whole particle.

The gas-phase concentration at equilibrium with the particle is assumed to correspond to the sum of the gas-phase concentrations at equilibrium with each layer weighted by their kinetic of diffusion:

$A_{\mathrm{g}, i}^{\mathrm{eq}, \text { bin }}=\sum_{\text {layer }}^{N_{\text {layer }}} f_{i}^{\text {bin,layer }} A_{\mathrm{g}, i}^{\text {eq,bin,layer }}$,

where $A_{\mathrm{g}, i}^{\mathrm{eq}, i n, \text { layer }}$ is the gas-phase concentration at equilibrium with the layer and with $f_{i}^{\text {bin,layer }}$ a weighting factor that depends on the kinetic of diffusion:

$A_{\mathrm{g}, i}^{\mathrm{eq}, \text { bin,layer }}=K_{\mathrm{effect}} \frac{A_{\mathrm{p}, i}^{\text {bin,layer }}}{K_{\mathrm{p}, i}^{\text {bin,layer }} M_{\mathrm{o}}^{\text {bin,layer }}}$, where $K_{\text {effect }}$ is the Kelvin effect correction coefficient computed with Eq. (47).

The coefficient $f_{i}^{\text {bin,layer }}$ corresponds to the fraction of the compound $i$ that arrives by diffusion in the layer if compound $i$ condenses or the fraction of the compound $i$ that departs from the layer if compound $i$ evaporates. The weighting factor is computed with the following algorithm, which separates positive fluxes from negative fluxes. In the case of condensation of compound $i\left(J_{\mathrm{tot}, i}^{\mathrm{bin}}>0\right)$, the compound condenses preferentially on layers with positive diffusion fluxes $J_{\text {diff, }, i}^{\text {biner }}$. Therefore, if $J_{\text {diff, } i}^{\text {biner }}$ is negative inside a layer, this layer is not taken into account in the condensation flux. On the other hand, in the case of evaporation of compound $i\left(J_{\mathrm{tot}, i}^{\mathrm{bin}}<0\right)$, the compound evaporates from layers with negative $J_{\text {diff, } i}^{\text {biner }}$. In other words, $f_{i}^{\text {bin,layer }}$ is computed by weighting the diffusion fluxes $J_{\mathrm{diff}, i}^{\text {bin,layer }}$ depending on their signs.

$f_{i}^{\text {bin,layer }}=\frac{J_{i}^{\text {bin,layer }}}{\sum_{\text {layer }}^{N_{\text {layer }}} J_{i}^{\text {bin,layer }}}$,

where $J_{i}^{\text {bin,layer }}$ is a flux that depends on the diffusion flux sign in each layer:

If the flux of diffusion of compound $i$ into the particle is positive $\left(J_{\mathrm{diff}, i}^{\mathrm{bin}}>0\right)$

when for the interface layer

$J_{i}^{\text {bin,layer }}=J_{i}^{\text {bin, interface }}$ if $J_{i}^{\text {bin,interface }}>0$ else $J_{i}^{\text {bin,layer }}=0$

and for other layers

$J_{i}^{\text {bin,layer }}=J_{\text {diff }, i}^{\text {bin,layer }}$ if $J_{\text {diff, } i}^{\text {bin,layer }}>0$ else $J_{i}^{\text {bin,layer }}=0$

If the flux of diffusion of compound $i$ into the particle is negative $\left(J_{\mathrm{diff}, i}^{\mathrm{bin}}<0\right)$

when for the interface layer

$J_{i}^{\text {bin,layer }}=J_{i}^{\text {bin,interface }}$ if $J_{i}^{\text {bin,interface }}<0$ else $J_{i}^{\text {bin,layer }}=0$

and for other layers

$J_{i}^{\text {bin,layer }}=J_{\text {diff, } i}^{\text {bin,layer }}$ if $J_{\text {diff, } i}^{\text {bin,layer }}<0$ else $J_{i}^{\text {bin,layer }}=0$ 
Once the weighting factors are computed, the flux of condensation-evaporation-diffusion over all layers $J_{\mathrm{tot}, i}^{\mathrm{bin}}$ can be computed with Eqs. (49)-(51). These weighting factors are used not only to redistribute the gas-phase concentration at equilibrium between layers from a bulk gas-phase concentration (Eq. 52), but also to redistribute the total flux of condensation-evaporation-diffusion between layers from the bulk total flux of condensation-evaporation-diffusion. The computation of the total flux per layer $J_{\text {tot, }, i}^{\text {biner }}$ is now explained. The total flux of condensation-evaporationdiffusion $J_{\text {tot }, i}^{\text {bin }}$ is separated into three fluxes $J_{i}^{+}$(equal to the sum of diffusion fluxes which are positive), $J_{i}^{-}$(equal to the sum of diffusion fluxes which are negative) and the flux at the interface $J_{i}^{\text {bin,interface }}$ :

$J_{\mathrm{tot}, i}^{\mathrm{bin}}=J_{i}^{\mathrm{bin}, \text { interface }}+J_{i}^{+}+J_{i}^{-}$.

By definition, $J_{i}^{+}$and $J_{i}^{-}$are equal to

$$
\begin{aligned}
& J_{i}^{+}=\sum_{\text {layer }}^{N_{\text {layer }}-1} \max \left(J_{\text {tot }, i}^{\text {bin,layer }}, 0\right), \\
& J_{i}^{-}=\sum_{\text {layer }}^{N_{\text {layer }}-1} \min \left(J_{\text {tot }, i}^{\text {bin,layer }}, 0\right),
\end{aligned}
$$

where $J_{\text {tot }, i}^{\text {bin,layer }}$ is the total flux of condensation-evaporationdiffusion of a compound $i$ inside a bin and a layer.

If the compound $i$ condenses into the particle $\left(J_{\mathrm{tot}, i}^{\mathrm{bin}}>\right.$ 0 ), we also have $J_{\text {cond/evap, } i}^{\text {bin }}>0$ for Eq. (51). In this case, if the diffusion flux $J_{\text {diff, } i}^{\text {biner }}$ is negative, then the compound moves by diffusion oppositely to condensation and therefore it should not be affected by the kinetic of condensation-evaporation. More generally, compounds and layers for which diffusion reacts in a different direction as condensation-evaporation (fluxes of different signs) should not be affected by the kinetic of condensation-evaporation (because the compounds are transferred from one phase to another without exchange with the gas phase). In other words,

if $J_{\mathrm{cond} / \mathrm{evap}, i}^{\mathrm{bin}} \times J_{\mathrm{diff}, i}^{\mathrm{bin}, \text { layer }}<0$ then $J_{\mathrm{tot}, i}^{\mathrm{bin}, \text { layer }}=J_{\mathrm{diff}, i}^{\mathrm{bin}, \text { layer }}$.

The layers with $J_{\text {cond/evap }, i}^{\text {bin }} \times J_{\text {diff, } i}^{\text {bin,layer }}<0$ are the layers with a diffusion flux sign opposite to $J_{\text {tot }, i}^{\mathrm{bin}}$. Therefore, the sum over these layers allows us to compute $J_{i}^{-}$with Eq. (58) if $J_{\mathrm{tot}, i}^{\mathrm{bin}}>0$ and $J_{i}^{+}$with Eq. (57) if $J_{\mathrm{tot}, i}^{\mathrm{bin}}<0$. If $J_{\mathrm{tot}, i}^{\mathrm{bin}}>0$ (resp. $J_{\text {tot }, i}^{\text {bin }}<0$ ), then $J_{i}^{+}$(resp. $J_{i}^{-}$) corresponds to the part of the total flux that is affected by both condensationevaporation and diffusion and $J_{i}^{-}$(resp. $J_{i}^{+}$) corresponds to the part of the total flux that is only affected by diffusion. $J_{i}^{-}$ is computed by Eqs. (59) and (58) (resp. $J_{i}^{+}$is computed by Eqs. 59 and 57), and $J_{i}^{+}$is deduced by Eq. (56).
The total positive (resp. negative) flux $J_{i}^{+}$(resp. $J_{i}^{-}$) is redistributed onto layers by using the weighting factors $f_{i}^{\text {bin,layer }}$

$J_{\text {tot }, i}^{\text {bin,layer }}=\frac{f_{i}^{\text {bin,layer }}}{\sum_{\text {layer }} f_{i}^{\text {bin,layer }}-f_{i}^{\text {bin,interface }}} J^{+}$

(if $J_{\text {tot, }, i}^{\text {bin,layer }}>0$ ),

$J_{\text {tot }, i}^{\text {bin,layer }}=\frac{f_{i}^{\text {bin,layer }}}{\sum_{\text {layer }} f_{i}^{\text {bin,layer }}-f_{i}^{\text {bin,interface }}} J^{-}$

(if $J_{\text {tot, }, i}^{\text {bin,layer }}<0$ ).

\subsubsection{Characteristic time to reach equilibrium with the gas phase}

The system of differential to solve Eq. (48) is stiff, as in the same layer/bin, some species reach equilibrium with the gas phase much quicker than others (Capaldo et al., 2000). To solve it efficiently, it is necessary to separately solve cases at equilibrium from other cases, which are solved dynamically. To determine the characteristic time to reach equilibrium with the gas phase, Eq. (48) is rewritten using the total concentrations and defining $F_{i}^{\text {bin,layer }}=A_{\mathrm{p}, i}^{\text {bin,layer }} / A_{\mathrm{p}, i}$ (if $A_{\mathrm{p}, i}^{\mathrm{bin}, \text { layer }}$ is different from zero):

$$
\begin{aligned}
\frac{\mathrm{d} A_{\mathrm{p}, i}^{\text {bin,layer }}}{\mathrm{d} t}=k & \left(A_{\mathrm{tot}, i} K_{\mathrm{p}, i}^{\text {bin,layer }} M_{\mathrm{o}}^{\text {bin,layer }}-A_{\mathrm{p}, i}^{\text {bin,layer }}\right. \\
& \left.\left(1+\frac{K_{\mathrm{p}, i}^{\text {bin,layer }} M_{\mathrm{o}}^{\text {bin,layer }}}{F_{i}^{\text {bin,layer }}}\right)\right) .
\end{aligned}
$$

This differential equation is solved by assuming that $F_{i}^{\text {bin,layer }}$ and $K_{\mathrm{p}, i}^{\text {bin,layer }} M_{\mathrm{o}}^{\text {bin,layer }}$ are constant (or do not vary much over one time step):

$$
\begin{aligned}
A_{\mathrm{p}, i}^{\text {bin,layer }}(t)=A_{\mathrm{eq}}+\left(A_{\mathrm{p}, i}^{\text {bin,layer }}(t=0)-A_{\mathrm{eq}}\right) \times \\
\exp \left(-\frac{t}{\tau_{\mathrm{eq}}}\right),
\end{aligned}
$$

where $A_{\mathrm{eq}}$ is the concentration at equilibrium with the gas phase and $\tau_{\text {eq }}$ the characteristic time to reach equilibrium.

$$
\begin{gathered}
A_{\mathrm{eq}}=\frac{A_{\mathrm{tot}, i} K_{\mathrm{p}, i}^{\text {bin,layer }} M_{\mathrm{o}}^{\text {bin,layer }}}{1+\frac{K_{\mathrm{p}, i}^{\text {bin,layer }} M_{\mathrm{o}}^{\text {bin,layer }}}{F_{i}^{\text {bin,layer }}}} \\
\tau_{\mathrm{eq}}^{-1}=\left(1+\frac{K_{\mathrm{p}, i}^{\text {bin,layer }} M_{\mathrm{o}}^{\text {bin,layer }}}{F_{i}^{\text {bin,layer }}}\right) k
\end{gathered}
$$

$\tau_{\text {eq }}$ is used to estimate the time necessary to reach equilibrium with the gas phase and therefore to identify cases that should be assumed at equilibrium when solving the system of Eq. (48). 


\subsubsection{Generalization to several organic phases}

The OM can be separated into several organic phases. Whereas the evolution of condensation-evaporation is dynamically modeled, phase separation and the repartition of compounds between organic phases are assumed to be at equilibrium. They are assumed to occur instantaneously: if an organic phase is saturated, it is divided instantaneously into several organic phases. The dynamic evolution due to condensation-evaporation in viscous particles is described by the following equation derived from Eq. (48) by taking into account the phase of the components:

$$
\begin{aligned}
& \frac{\mathrm{d} A_{\mathrm{p}, i}^{\text {bin,layer,phase }}}{\mathrm{d} t}=J_{\text {tot, } i}^{\text {bin,layer,phase }}= \\
& \frac{K_{\mathrm{p}, i}^{\text {bin,layer,phase }} M_{\mathrm{O}}^{\text {bin,layer,phase }}}{\sum_{\phi}^{N_{\text {phase }}} K_{\mathrm{p}, i}^{\text {bin,layer, } \phi} M_{\mathrm{O}}^{\text {bin,layer, } \phi}} J_{\text {tot }, i}^{\text {bin,layer }},
\end{aligned}
$$

where $J_{\text {tot, } i}^{\text {bin,layer,phase }}$ is the flux of condensation-evaporationdiffusion into an organic phase. $J_{\text {tot, }, i}^{\text {bin,layer }}$ is computed with the system of Eqs. (40)-(61) generalized to several organic phases. Equation (40) is generalized into

$$
\begin{aligned}
\frac{J_{\text {diff }}^{\text {bin,layer }}}{\mathrm{d} t} & =\sum_{\text {phase }}^{N_{\text {phase }}} \frac{J_{\text {diff }}^{\text {bin,layer,phase }}}{\mathrm{d} t} \\
& =\sum_{\text {phase }}^{N_{\text {phase }}} f_{\text {morph }}^{\text {layer }} k_{\text {diffusion }} \\
& \left(A_{\mathrm{g}, i} K_{\mathrm{p}, i}^{\text {bin,layer }, i \text { phase }} M_{\mathrm{o}}^{\text {bin,layer, } i \text { phase }}-A_{\mathrm{p}, i}^{\text {bin,layer }, i \text { phase }}\right),
\end{aligned}
$$

where $M_{\mathrm{o}}^{\text {bin,layer,phase }}$ is the total organic concentrations in the specified organic phase in a specified bin and layer (in $\mu \mathrm{g} \mathrm{m}^{-3}$ ), $J_{\text {diff }}^{\text {bin,layer,phase }}, A_{\mathrm{p}, i}^{\text {bin,layer,phase }}$ and $K_{\mathrm{p}, i}^{\text {bin,layer,phase }}$ the flux of condensation-evaporation-diffusion, concentration and partitioning coefficient of the compound $i$ for a bin, a layer and a phase, respectively. The flux at the interface is computed via Eq. (44) using the gas-phase concentration at equilibrium with the interface:

$$
\begin{aligned}
& A_{\mathrm{g}, i}^{\mathrm{eq}, \text { bin,interface }}= \\
& \quad K_{\text {effect }} \frac{\sum_{\text {phase }}^{N_{\text {phase }}} A_{\mathrm{p}, i}^{\text {bin,interface,phase }}}{\sum_{\text {phase }}^{N_{\text {phase }}} K_{\mathrm{p}, i}^{\text {bin,interface,phase }} M_{\mathrm{o}}^{\text {bin,interface,phase }}} .
\end{aligned}
$$

The flux of condensation-evaporation over the whole particle is computed via Eq. (51) by using the gas-phase concentration at equilibrium with a layer:

$$
A_{\mathrm{g}, i}^{\mathrm{eq}, \text { bin,layer }}=\frac{\sum_{\mathrm{phase}}^{N_{\text {phase }}} A_{\mathrm{p}, i}^{\text {bin,layer,phase }}}{\sum_{\text {phase }}^{N_{\text {phase }}} K_{\mathrm{p}, i}^{\text {bin,layer,phase }} M_{\mathrm{o}}^{\text {bin,layer,phase }}} .
$$

At each time step, thermodynamic evolution is first computed. The number of organic phases and the distribution of compounds between organic phases are then computed for each layer and each bin by assuming equilibrium between phases. To compute the concentrations with several organic phases at equilibrium we first study the conditions that have to be respected. The first condition is that the activities of each compound $i$ are equal in each phase. For example, for two phases phase ${ }_{1}$ and phase $_{2}$ :

$$
\begin{aligned}
& \gamma_{i}^{\text {bin,layer,phase }}{ }_{i}^{\text {bin,layer,phase }}{ }^{\text {bing }}= \\
& \gamma_{i}^{\text {bin,layer,phase }}{ }^{\text {bin,layer,phase }} x_{i} .
\end{aligned}
$$

The second condition, which is that each phase may be at equilibrium with the gas-phase (if condensation is too quick to be solved dynamically), can be written as

$$
\begin{aligned}
& \frac{A_{\mathrm{p}, i}^{\text {bin,layer,phase }}{ }_{1}}{A_{\mathrm{p}, i}^{\text {bin,layer,phase }}{ }_{2}}=\frac{A_{\mathrm{p}, i}^{\text {bin,layer,phase }}{ }_{1}}{A_{\mathrm{g}}} \times \frac{A_{\mathrm{g}}}{A_{\mathrm{p}, i}^{\text {bin,layer,phase }}{ }_{2}}= \\
& \frac{K_{\mathrm{p}, i}^{\text {bin,layer,phase }_{1}} M_{\mathrm{O}}^{\text {bin,layer,phase }}{ }}{K_{\mathrm{p}, i}^{\text {bin,layer,phase }_{2}} M_{\mathrm{O}}^{\text {bin,layer,phase }}{ }_{2}} .
\end{aligned}
$$

To respect these two conditions, the Kelvin effect must be the same for each phase.

$$
\begin{gathered}
\left(\exp \left(-\frac{2 \sigma M_{\text {surf }}^{\text {bin }}}{\operatorname{RT} \rho_{\text {organic }} R_{\mathrm{p}}}\right)\right)^{\text {phase }_{1}}= \\
\left(\exp \left(-\frac{2 \sigma M_{\text {surf }}^{\text {bin }}}{\text { RT } \rho_{\text {organic }} R_{\mathrm{p}}}\right)\right)^{\text {phase }_{2}} .
\end{gathered}
$$

$M_{\text {surf }}^{\text {bin }}, \rho_{\text {organic }}$ and $\sigma$ must be the same in the two phases.

Therefore, if there are several organic phases, the partitioning coefficient must be computed with the following equation

$$
\begin{aligned}
& K_{\mathrm{p}, i}^{\text {bin,layer,phase }}=\frac{760 \times 8.202 \times 10^{-5} \times T}{M_{\mathrm{ow}}^{\text {bin,layer,phase }} \gamma_{i, \text { org }}^{\text {bin,layer,phase }} P_{i}^{0} \times 10^{6}} \\
& \quad \exp \left(-\frac{2 \sigma M_{\text {surf }}^{\text {bin }}}{\mathrm{RT} \rho_{\text {organic }} R_{\mathrm{p}}}\right),
\end{aligned}
$$

where $M_{\text {surf }}^{\text {bin }}$ is the mean molar mass of all organic phases at the surface of particles.

$$
M_{\mathrm{surf}}^{\mathrm{bin}}=\frac{\sum_{i} \sum_{\text {phase }} A_{\mathrm{p}, i}^{\text {bin,surface,phase }}}{\sum_{i} \sum_{\text {phase }} \frac{A_{\mathrm{p}, i}^{\text {bin,surface,phase }}}{M_{i}}}
$$

The characteristic times are assumed to be the same for all the organic phases to prevent a compound from being absorbed dynamically into an organic phase when it is at equilibrium with another organic phase. The characteristic time 
Table 2. Polynomial parameters for Eq. (41).

\begin{tabular}{lccccc}
\hline & Layer & $A_{\text {layer }}$ & $B_{\text {layer }}$ & $C_{\text {layer }}$ & $D_{\text {layer }}$ \\
\hline \multirow{3}{*}{ Three diffusion layers } & 1 & 0.5294 & -1.6357 & 2.2070 & -2.1006 \\
& 2 & -0.3171 & 0.8786 & -1.0613 & -0.5002 \\
& 3 & 0.1375 & -0.3707 & 0.4698 & -1.2367 \\
\hline \multirow{3}{*}{ Four diffusion layers } & 1 & 0.6880 & -1.9700 & 2.4440 & -2.1617 \\
& 2 & 0.1210 & 0.3567 & -1.1614 & -0.2698 \\
& 3 & 0.3155 & -0.7826 & 0.7822 & -1.3147 \\
& 4 & -0.1364 & 0.2046 & 0.3282 & -1.3954 \\
\hline
\end{tabular}

is then computed according to the following equation derived from Eq. (65).

$$
\begin{gathered}
\tau_{\text {eq }}^{\text {bin,layer,phase }}= \\
\frac{1}{f_{\text {morph }}^{\text {layer }} k_{\text {diffusion }}}+\frac{\sum_{\text {phase }} K_{\mathrm{p}, i}^{\text {bin,layer,layer }} M_{\mathrm{o}}^{\text {bin,layer,layer }}}{\sum_{\text {phase }} K_{\mathrm{p}, i}^{\text {bin,layer,phase }} M_{\mathrm{o}}^{\text {bin,layer,phase }}} \\
f_{i}^{\text {bin,layer }}
\end{gathered}
$$

\subsubsection{Absorption into the aqueous phase}

For concentrations in the organic phases, the dynamic evolution follows Eq. (66) but the dynamic evolution of concentrations in the aqueous phase follows Eq. (76), because condensation-evaporation is assumed to not be limited by diffusion in the aqueous phase and a multi-layer representation is then not useful.

$$
\frac{\mathrm{d} A_{\mathrm{aq}, i}^{\mathrm{bin}}}{\mathrm{d} t}=k_{\mathrm{absorption}}\left(A_{\mathrm{g}, i}-\frac{A_{\mathrm{aq}, i}^{\mathrm{bin}}}{K_{\mathrm{aq}, i}^{\mathrm{bin}} \mathrm{AQ}^{\mathrm{bin}}}\right)
$$

\subsubsection{Absorption into a particle with an aqueous phase and organic phases}

Under most atmospheric conditions, particles are probably not entirely organic or entirely aqueous. The surface of particles is probably covered partially by both the OM and by the aqueous phase. Equations (76) and (66) are still valid but $k_{\text {absorption }}$ in Eqs. (76) and (45) must be corrected to take into account that there is a chance that a compound trying to condense into a phase encounters the wrong phase, i.e., a phase into which it may not condense.

The chance for a compound to encounter an aqueous phase fsurf $_{\mathrm{aq}}$ is computed with Eq. (77)

$\operatorname{fsurf}_{\mathrm{aq}}=\frac{S_{\mathrm{aq}}}{S_{\mathrm{tot}}}$,

where $S_{\mathrm{aq}}$ is the surface of particles that is aqueous and $S_{\text {tot }}$ the total surface of particles.
To evaluate this parameter, we assume that the surface of the particle is only covered by the aqueous phase and the organic phases and that the ratio of the aqueous surface over the organic surface is equal to the ratio of the volume of the aqueous phase over the volume of the organic phases:

$$
\begin{aligned}
& \text { fsurf }_{\mathrm{aq}}=\frac{V_{\mathrm{aq}}}{V_{\mathrm{aq}}+V_{\mathrm{org}}}= \\
& \frac{\frac{\mathrm{AQ}_{\text {aqueous }}^{\text {bin }}}{\rho_{\text {aqueou }}}}{\frac{\mathrm{AQ}^{\text {bin }}}{\rho_{\text {aqueous }}}+\sum_{\text {layer }}^{\text {Nlayer }} \sum_{\text {phase }}^{\text {Nphase }} \frac{M_{\mathrm{o}}^{\text {bin,layer,phase }}}{\rho_{\text {organic }}}} .
\end{aligned}
$$

For the condensation in the aqueous phase, by taking into account the chance for a compound to encounter the aqueous phase, $k_{\text {absorption }}$ is

$k_{\text {absorption }}=$ fsurf $_{\mathrm{aq}} \times 2 \pi D_{\mathrm{p}} D_{\text {air }} N^{\text {bin }} f(K n, \alpha)$.

For the condensation in the organic phases, by taking into account the chance for a compound to encounter the organic phases, $k_{\text {absorption }}$ is

$k_{\text {absorption }}=\left(1.0-\right.$ fsurf $\left._{\mathrm{aq}}\right) \times 2 \pi D_{\mathrm{p}} D_{\text {air }} N^{\text {bin }} f(K n, \alpha)$.

\subsubsection{Redistribution of compounds between layers}

To use the approach described in this paper, the mass fraction (ratio of the mass of the layer over the mass of the particle) of layers must stay constant throughout the simulation and the mass of each layer must respect the condition given by Eq. (37), which specifies the mass of the layer with respect to the total mass of the particle. However, due to rapid condensation or evaporation of the layer near the interface, concentrations of organic compounds may need to be redistributed over layers to respect this condition. If compounds are not redistributed some layers may become larger (due to the differences in fluxes) and the layer near the interface may for example become larger than the layer at the center of the particle. Moreover, as the volume of the particle changes with condensation-evaporation, the concentration of a layer can be transferred to other layers. If a particle grows due to condensation into the layer at the interface, compounds that were previously in the interface layer are pushed into more internal layers and the newly condensed compounds remain at the 
Table 3. Algorithm to compute the redistribution of compounds between layers.

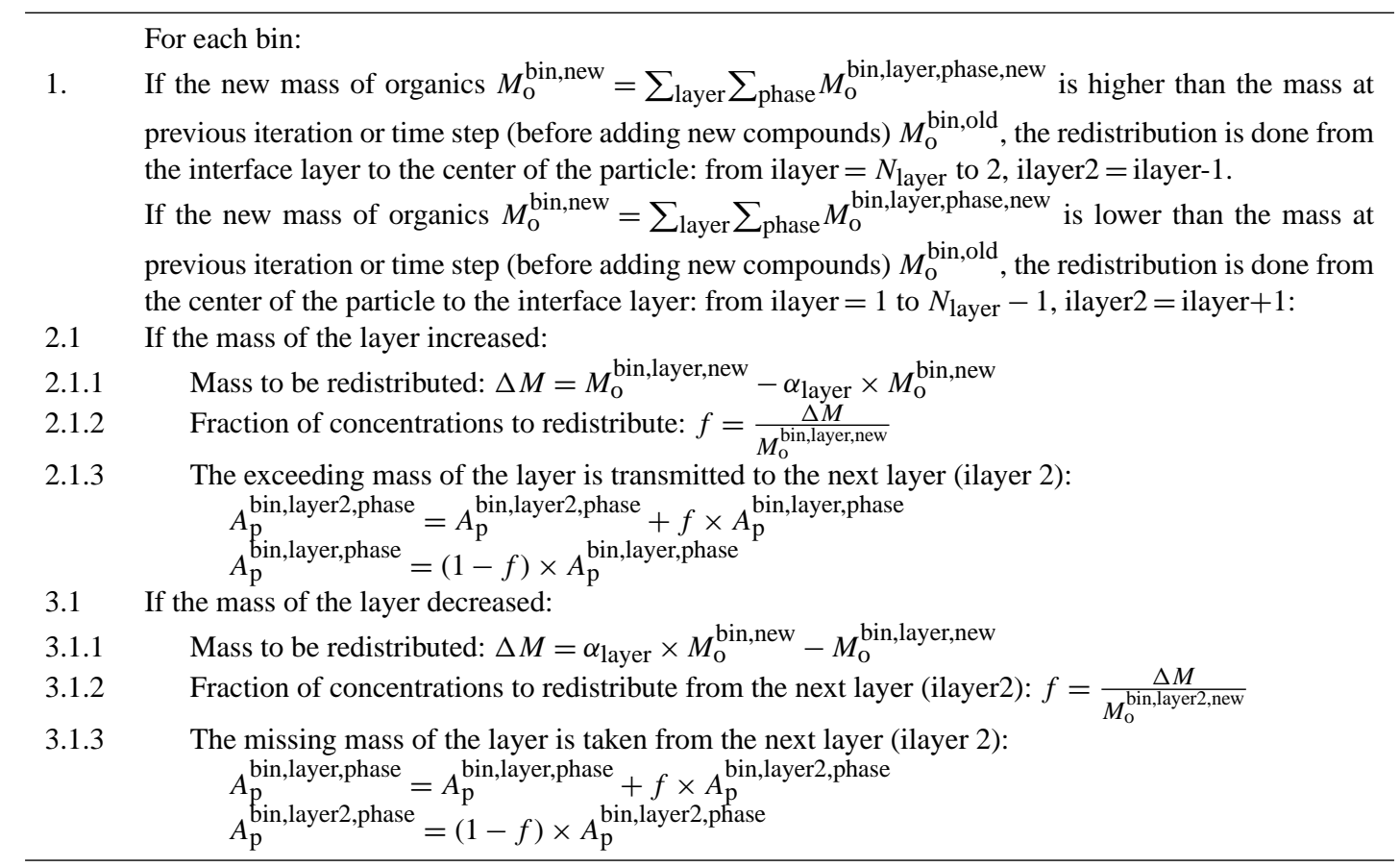

interface layer. On the other hand, if a particle shrinks due to evaporation of the layer at the interface, the missing mass of the layer will be taken from more internal layers. For a case of evaporation, the mass of the layer at the interface may be too low (due to the more rapid evolution at the interface) and the missing mass of the layer is taken from layers at the inside of the particle, i.e., concentrations are redistributed from the outside to the inside. For a case of condensation, the mass of the layer at the interface may be too high, the exceeding mass of the layer is redistributed over the layers at the inside of the particle, i.e., concentrations are redistributed from the inside to the outside. The algorithm is detailed in Table 3.

The redistribution algorithm that creates numerical diffusion as a small fraction of the mass of a layer is always transferred to other layers. This redistribution effect should decrease if the number of layers increases but it is necessary for a 3-D application to keep the number of layers low. However, the redistributed amount should be low compared to the absorbed amount of organic compounds. Similarly, in a 3-D air quality model, concentrations and number of particle in size sections have to be redistributed between sections so that the bounds diameters of sections are kept constant, which does also create some numerical issues. In SOAP, the diameters of sections evolve according to the mass that condenses or evaporates (without changing the number in sections) and there is no size redistribution between sections. However, a size-section redistribution algorithm should be added to the code if coagulation is added (for modeling purposes) or in 3-
D models (in which SOAP would be implemented) after the call of SOAP.

\subsubsection{Thermodynamic equilibrium}

For numerical stability, some compounds in some bins and layers are assumed to be at equilibrium with the gas phase because equilibrium is reached very quickly (for example for very volatile compounds). To identify cases where equilibrium with the gas phase should be assumed, a criterion $t_{\text {equilibrium }}$ is used. If the characteristic time to reach equilibrium with the gas phase is lower than $t_{\text {equilibrium, the case }}$ is considered at equilibrium, whereas if it is higher than $t_{\text {equilibrium, }}$ the case is solved dynamically.

Concentrations of organic compounds in the organic phases are computed according to Eq. (81) (obtained by generalizing Eq. 2 for several bins, layers and phases):

$$
A_{\mathrm{p}, i}^{\text {bin,layer,phase }}=\operatorname{conc}_{i, \mathrm{eq}} \frac{K_{\mathrm{p}, i}^{\text {bin,layer,phase }} M_{\mathrm{o}}^{\text {bin,layer,phase }}}{1+\text { ratio }_{i, \mathrm{eq}}},
$$

where conc $_{i, \text { eq }}$ is the total concentration of compound $i$ at equilibrium with the gas phase (sum of the gas-phase concentration and of organic-phase concentrations in layers and bins at equilibrium) computed with Eq. (82) and ratio $_{i \text {, eq }}$ the ratio of the concentration of compound $i$ at equilibrium with the gas phase in the particle phase to the concentration of $i$ in the gas phase computed with Eq. (83) (similar to Eq. 15 for several bins, layers and phases).

$$
\text { conc }_{i, \mathrm{eq}}=A_{\mathrm{tot}, i}-\sum_{\text {bin layerphase }} \sum\left(1-\lambda^{\text {bin,layer,phase }}\right) A_{\mathrm{p}, i}^{\text {bin,layer,phase }}
$$




$$
\begin{aligned}
& \text { ratio }_{i, \text { eq }}= \frac{\sum_{\text {bin layerphase }} \sum_{\text {bin,layer,phase }} A_{\mathrm{p}, i}^{\text {bin,layer,phase }}}{A_{\mathrm{g}}} \\
&=\sum_{\text {bin layerphase }} \sum \lambda^{\text {bin,layer,phase }} K_{\mathrm{p}, i}^{\text {bin,layer,phase }} M_{\mathrm{o}}^{\text {bin,layer,phase }}
\end{aligned}
$$

With $\lambda^{\text {bin,layer,phase }}$ defined such as

$$
\begin{aligned}
\lambda^{\text {bin,layer,phase }} & =1 \quad \text { if } \tau_{\text {eq }}^{\text {bin,layer,phase }}<t_{\text {equilibrium }} \text { (case at equilibrium) } \\
& =0 \quad \text { if } \tau_{\text {eq }}^{\text {bin,layer,phase }}>=t_{\text {equilibrium }} \text { (dynamic case). }
\end{aligned}
$$

The system is solved iteratively, as now detailed. The composition of the particles is first estimated using Eqs. (81)(84). If the concentrations computed from this estimation are different from those obtained in the previous iteration, a new estimation of concentrations is computed. The algorithm is detailed in Table 1. Step 7 and step 2 prevent the non-convergence due to high variations of concentrations by reducing the variations between two iterations.

Some numerical issues can arise from the equilibrium representation especially for low volatility compounds with

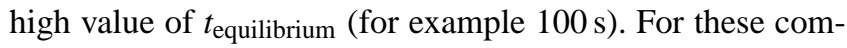
pounds, assuming equilibrium with the gas phase will give errors because these compounds will condense almost entirely on the bin with the higher organic mass instead of condensing on each bin according to the kinetics of condensation. To prevent this problem, low volatility cases (with $K_{\mathrm{p}, i}^{\text {bin,layer,phase }}>10$ ) are assumed dynamic.

\subsubsection{Methodology used to compute the evolution of concentrations}

The method used to solve the evolution of concentrations is shown in Fig. 5. As mentioned in the previous section, the model uses a hybrid method combining a dynamic representation where concentrations evolve as a function of time (for cases with characteristic times higher than a $t_{\text {equilibrium value }}$ specified by the user) with an equilibrium representation (for cases with characteristic times lower than the $t_{\text {equilibrium }}$ value). In the equilibrium representation, the distribution of organic compounds between phases and the gas/particle partitioning of cases with characteristic times lower than $t_{\text {equilibrium }}$ are computed.

In the dynamic representation, concentrations evolving dynamically (cases with characteristic times higher than $t_{\text {equilibrium }}$ ) are computed as a function of the time step with the second-order Rosenbrock scheme ROS2 (Verwer et al., 1999) for time integration. The time step can be rejected (the computation is redone with another time step) if the error between the second-order and the first-order concentrations is too high (higher than an EPSER parameter specified by the user). In that case, the time step decreases until the error is lower than EPSER (until the time step is accepted). If the time step is very low, the ROS2 scheme increases the time step to obtain the optimal time step.

To compute concentrations, the model first initializes required properties (activity coefficients, the number of phases in each layer and the characteristic times). For the first time step, the model calls for the first time the equilibrium representation. Once the equilibrium is reached, the model computes the dynamic evolution with the dynamic representation. Because concentrations changed, the gas/particle partitioning of cases at equilibrium changed, the equilibrium representation is then called again. For the next time steps, the dynamic and equilibrium representations are called once each, until the ending time of the simulation is reached.

\section{Results}

In this section, the dynamic implicit representation of SOAP is first compared to an explicit representation to check that the dynamic evolution of surrogates between the gas and particle phases is well represented by the implicit representation. Two test cases representative of European summer periods with high biogenic concentrations and high anthropogenic concentrations are then defined. Simulations are performed to study the impact of ideality, saturation, phase separation and thermodynamic equilibrium on the model results. Comparisons of the implicit and explicit representations for one of the test case are also performed.

\subsection{Validation of the dynamic implicit representation}

To validate the dynamic implicit representation of SOAP, we study the condensation of an hydrophobic organic surrogate of different volatilities (with a partitioning coefficient of 100 , 1 , or $0.01 \mathrm{~m}^{3} \mu \mathrm{g}^{-1}$ ) for different organic-phase diffusion coefficients $\left(10^{-19}, 10^{-20}, 10^{-21}, 10^{-22}\right.$ and $\left.10^{-24} \mathrm{~m}^{2} \mathrm{~s}^{-1}\right)$ into $5 \mu \mathrm{g} \mathrm{m}^{-3}$ of non-volatile organic compounds. The organic surrogate that condenses is initially only in the gas phase, with a mass of $5 \mu \mathrm{g} \mathrm{m}^{-3}$. The size distribution of particles is divided into 5 bins. The number of particles inside each bin is assumed to be equal to $3.02 \times 10^{8}, 1.40 \times 10^{9}$, $2.24 \times 10^{8}, 1.69 \times 10^{6} ; 12224.0$ particles $\mathrm{m}^{-3}$ based on average simulation results over Europe (Couvidat et al., 2012). The initial mass concentrations of the surrogate are distributed between each section with the following fraction: $0.7,26.6,61.4,11.2$ and $0.1 \%$. The initial diameter of each bin is computed from the initial mass and number concentrations in the bin.

The results of the implicit representation are compared with an explicit representation where diffusion between layers is treated explicitly. In the explicit representation, the diffusion of organic compounds in the particle is represented explicitly as in KM-GAP (with no equilibrium assumption). The particles are discretized with 100 layers, each of them having a volume representing $1 \%$ of the total volume of the 

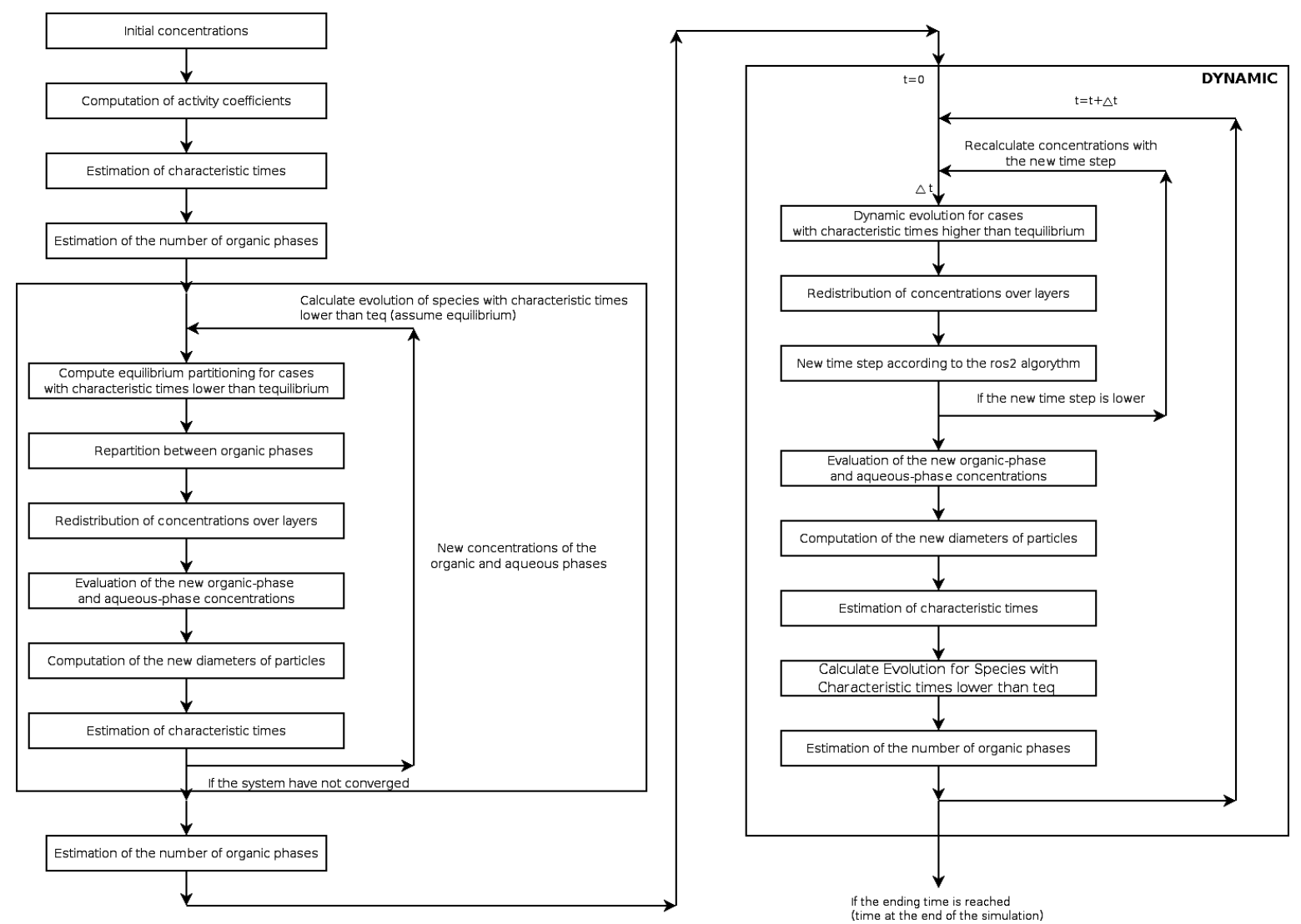

Figure 5. Diagram of the method used to compute the evolution of concentrations.

particles. The evolution of concentrations inside each layer $i$ is computed by

$C_{i}(t+\Delta t)=C_{i}(t)+\frac{F_{i, i+1}+F_{i, i-1}}{V_{\text {layer }}} \Delta t$

where $F_{i, i+1}$ and $F_{i, i-1}$ are the fluxes of diffusion between the layer $i$ and the nearby layers (the computation of those fluxes is given in the Supplement) and $V_{\text {layer }}$ the volume of the layer. As in the implicit representation, the volume of each layer is kept constant throughout the simulation using the redistribution scheme presented in Sect. 2.3.8.

Figure 6 shows the comparison between the explicit representation with 100 layers and the implicit representation with four and five layers (one of these layer being the interface layer not affected by diffusion). The implicit representation reproduces the result of the explicit representation well. For non-volatile compounds (with a partitioning coefficient around $100 \mathrm{~m}^{3} \mu \mathrm{g}^{-1}$ ), there is no significant differences between the two methods. For such compounds, the condensation is not limited by diffusion and therefore they condense independently of the organic-phase diffusion coefficient. For low volatility compounds (with a partitioning coefficient around $1 \mathrm{~m}^{3} \mu \mathrm{g}^{-1}$ ), first, condensation into the first layer occurs without a limitation due to diffusion and then diffusion over all the particle occurs. The implicit representation with 5 layers slightly underestimates the diffusion of low volatility compounds for low organic-phase diffusion coefficients $\left(10^{-19} \mathrm{~m}^{2} \mathrm{~s}^{-1}\right)$ but reproduces very well cases with lower diffusion coefficients (lower than $10^{-20} \mathrm{~m}^{2} \mathrm{~s}^{-1}$ ), whereas the implicit representation with 4 layers slightly overestimates the diffusion of low volatility compounds. For more volatile compounds (with a partitioning coefficient around $0.01 \mathrm{~m}^{3} \mathrm{\mu g}^{-1}$ ), the implicit representation reproduces the explicit representation well with both four and five layers but underestimates low concentrations (lower than $10 \%$ of the concentration at equilibrium) with four layers.

To fully validate the implicit representation, a comparison of the implicit and explicit representations is also performed for one of the test cases (see Sect. 3.5).

\subsection{Setup of the test cases}

The behavior of the model is tested using two test cases. The first test case corresponds to a summer period with high concentrations of biogenic SOA, and the second test case corresponds to a summer period with high concentrations of anthropogenic SOA. These two test cases hereafter are referred as the biogenic test case and the anthropogenic test case. For the biogenic and the anthropogenic test cases, pollutant mass 

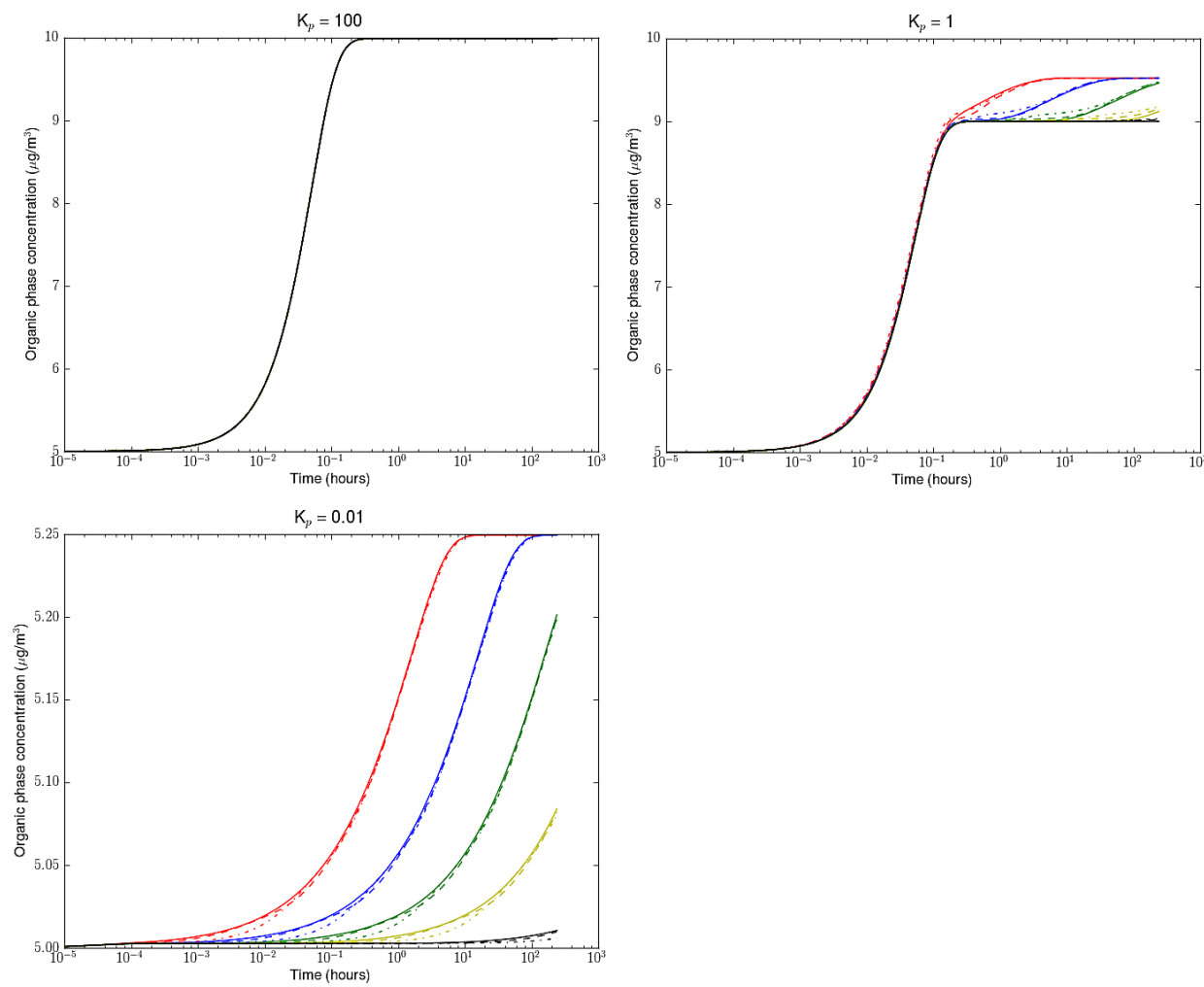

Figure 6. Comparison between the explicit representation (plain lines) and the implicit representation for $N_{\text {layer }}=5\left(-\right.$ line) and $N_{\text {layer }}=$ 4 (-. line) for several organic-phase diffusivity: $D_{\text {org }}=10^{-19} \mathrm{~m}^{2} \mathrm{~s}^{-1}$ (red), $D_{\text {org }}=10^{-20} \mathrm{~m}^{2} \mathrm{~s}^{-1}$ (blue), $D_{\text {org }}=10^{-21} \mathrm{~m}^{2} \mathrm{~s}^{-1}$ (green), $D_{\text {org }}=10^{-22} \mathrm{~m}^{2} \mathrm{~s}^{-1}$ (yellow) and $D_{\text {org }}=10^{-24} \mathrm{~m}^{2} \mathrm{~s}^{-1}$ (black) for a compound (with a partitioning coefficient of 100,1 and $0.01 \mu \mathrm{g} \mathrm{m}{ }^{-3}$ ) condensing on $5 \mu \mathrm{g} \mathrm{m}^{-3}$ of non-volatile organic compounds.

concentrations and meteorological conditions are extracted from the simulations of Couvidat et al. $(2012,2013)$ from over Europe and the Paris area, respectively.

The surrogates are the same as in the aerosol model $\mathrm{H}^{2} \mathrm{O}$, which was used by Couvidat et al. (2012, 2013). Surrogates are described in Table 4 and their total concentrations (gas + particle) are given in Table 5 for both test cases. For both test cases, the liquid water content of aerosol, the $\mathrm{pH}$, the ionic strength and the concentrations of inorganic ions are computed with the ISORROPIA model (Nenes et al., 1998) at a specified $\mathrm{RH}$. In $\mathrm{H}^{2} \mathrm{O}$, the surrogates that are representative of primary and aged SVOCs (POAlP, POAmP, POAhP, SOAIP, SOAmP and SOAhP) do not have a molecular structure attached. Therefore, processes depending on the molecular structure (like absorption on the aqueous phase) are not estimated for those surrogates. They are assumed to be hydrophobic and their influence on the activity coefficients of other species is taken into account by assigning to them a default molecular structure representative of primary aerosol and lowly oxidized compounds. This default structure consists of $40 \%$ of $\mathrm{C}_{23} \mathrm{H}_{47} \mathrm{COOH}$, $5 \%$ of $\mathrm{C}_{8} \mathrm{H}_{17} \mathrm{CH}=\mathrm{CHC}_{7} \mathrm{H}_{14} \mathrm{COOH}, 15 \%$ of 4-(2-propio)syringone, $12 \%$ of $\mathrm{C}_{29} \mathrm{H}_{60}$ and $28 \%$ of 2-carboxybenzoic acid based on EPRI (1999). In our test cases, the same default structure as in $\mathrm{H}^{2} \mathrm{O}$ is used.

The size distribution and the number of particles are the same as those used for the validation. The volume of the solid core of each size section is computed from the mass concentrations of solid species (dust, black carbon and solid inorganic are given by ISORROPIA) to compute morphology factors.

\subsection{Equilibrium representation}

To study the impact of activity coefficients, hydrophilic properties of surrogates, saturation and phase separation, concentrations in the particle phase are assumed to be at equilibrium with the gas phase for all surrogates.

\section{Influence of activity coefficients on organic aerosol formation (without phase separation of the organic phase)}

The effect of non-ideality on aerosol concentrations is strong and complex. To determine the impact of non-ideality, Tables 6 and 7 show the concentrations of organic aerosol for both test cases formed from the various precursors as well 
Table 4. Description of the $\mathrm{H}^{2} \mathrm{O}$ surrogate SOA species. Volatility is very low for compounds with $K_{\mathrm{p}}>100$, low for compounds with $100 \geq K_{\mathrm{p}}>1$, medium for compounds with $1 \geq K_{\mathrm{p}}>0.1$, high for compounds with $0.1 \geq K_{\mathrm{p}}>0.01$ and very high for compounds with $K_{\mathrm{p}} \leq 0.01$. A and B species are, respectively, hydrophilic and hydrophobic compounds.

\begin{tabular}{|c|c|c|c|c|}
\hline Surrogate & Type & Precursors & Conditions of formation & Volatility \\
\hline BiMT & A & isoprene & Oxidation by $\mathrm{OH}$ (low $\mathrm{NO}_{x}$ ) & high \\
\hline BiPER & A & isoprene & Oxidation by $\mathrm{OH}\left(\right.$ low $\mathrm{NO}_{x}$ ) & high \\
\hline BiDER & A & isoprene & Oxidation by $\mathrm{OH}$ (low $\mathrm{NO}_{x}$ ) & medium \\
\hline BiMGA & A & isoprene & Oxidation by $\mathrm{OH}\left(\right.$ high $\mathrm{NO}_{x}$ ) & medium \\
\hline BiNGA & $\mathrm{B}$ & isoprene & Oxidation by $\mathrm{OH}\left(\right.$ high $\mathrm{NO}_{x}$ ) & medium \\
\hline BiNIT3 & B & isoprene & Oxidation by $\mathrm{NO}_{3}$ & high \\
\hline BiA0D & A & monoterpenes & Oxidation by $\mathrm{OH}$ and $\mathrm{O}_{3}$ & $\begin{array}{l}\text { very low if the aqueous aerosol } \\
\text { is acidic }\end{array}$ \\
\hline BiA1D & A & monoterpenes & Oxidation by $\mathrm{OH}$ and $\mathrm{O}_{3}$ & medium \\
\hline $\mathrm{BiA} 2 \mathrm{D}$ & A & monoterpenes & Oxidation by $\mathrm{OH}$ and $\mathrm{O}_{3}$ & medium \\
\hline BiNIT & B & monoterpenes & Oxidation by $\mathrm{NO}_{3}$ & high \\
\hline $\mathrm{BiBlP}$ & B & sesquiterpenes & Oxidation by $\mathrm{OH}$ & very low \\
\hline $\mathrm{BiBmP}$ & $\mathrm{B}$ & sesquiterpenes & Oxidation by $\mathrm{OH}$ & medium \\
\hline AnBIP & $\mathrm{B}$ & aromatics & Oxidation by $\mathrm{OH}\left(\right.$ low $\mathrm{NO}_{x}$ ) & low \\
\hline $\mathrm{AnBmP}$ & $\mathrm{B}$ & aromatics & Oxidation by $\mathrm{OH}\left(\right.$ low $\mathrm{NO}_{x}$ ) & high \\
\hline $\mathrm{AnClP}$ & B & aromatics & Oxidation by $\mathrm{OH}$ (high $\mathrm{NO}_{x}$ ) & non-volatile \\
\hline POAIP & B & - & Primary SVOC & low \\
\hline POAmP & B & - & Primary SVOC & high \\
\hline POAhP & B & - & Primary SVOC & very high \\
\hline SOAlP & $\mathrm{B}$ & POAlP & Oxidation by $\mathrm{OH}$ & very low \\
\hline SOAmP & B & POAmP & Oxidation by $\mathrm{OH}$ & low \\
\hline SOAhP & $\mathrm{B}$ & POAhP & Oxidation by $\mathrm{OH}$ & high \\
\hline
\end{tabular}

as the concentrations of water, with and without the ideality assumption, respectively. The compounds are assumed to be both hydrophilic and hydrophobic except for the species POAIP, POAmP, POAhP, SOAIP, SOAmP and SOAhP, which are simply assumed to be hydrophobic, with the default molecular structure used in Couvidat et al. (2012).

Concentrations of compounds in the organic phase tend to decrease strongly when non-ideality is assumed (except for aromatics in the biogenic case due to non-linear effects), especially for the compounds formed from isoprene oxidation (most of them are very hydrophilic and therefore have low affinity with very hydrophobic compounds) and for some of the compounds formed from monoterpenes. Concentrations of hydrophilic compounds in the aqueous phase either increase or decrease depending on conditions. For the compounds formed from isoprene oxidation, their concentrations increase in the biogenic case from $0.70 \mu \mathrm{g} \mathrm{m}^{-3}$ for ideality to $1.24 \mu \mathrm{g} \mathrm{m}^{-3}$ for non-ideality with long-, medium- and short-ranges interactions and to $1.29 \mu \mathrm{g} \mathrm{m}^{-3}$ for non-ideality with only short-range interactions. It seems to indicate that in this case short-range interactions between organic compounds tend to stabilize hydrophilic organic compounds in the aqueous phase, whereas medium-range and long-range interactions between organic and inorganic compounds tend to destabilize hydrophilic organic compounds. These results are consistent with the result of Couvidat et al. (2012) where assuming ideality at infinite dilution can lead to an underestimation of the concentrations of hydrophilic species inside the aqueous phase of particles. Therefore, the concentrations of hydrophilic organic compounds in the aqueous phase probably depend strongly on other compounds and on the nonideality of aerosols.

\subsection{Hydrophobic versus hydrophilic}

Determining a priori if a compound is hydrophilic or hydrophobic is not straightforward. Table 8 shows that some compounds seem clearly hydrophobic (BiA2D, BiA1D, AnBlP, BiBlP, BiBmP, AnClP, BiNGA, BiNIT3, BiNIT) or hydrophilic (BiA0D) as they partition only into one phase. However, some compounds are present in both the organic and aqueous phases. AnBmP seems to be both hydrophilic and hydrophobic and can change phase depending on conditions. Moreover, BiA2D, BiA1D and BiMGA were assumed to be hydrophilic in $\mathrm{H}^{2} \mathrm{O}$ whereas it seems from these test cases that they are mainly hydrophobic. The fact that these compounds were assumed hydrophilic is probably due the choice of a criterion not representative of all atmospheric 


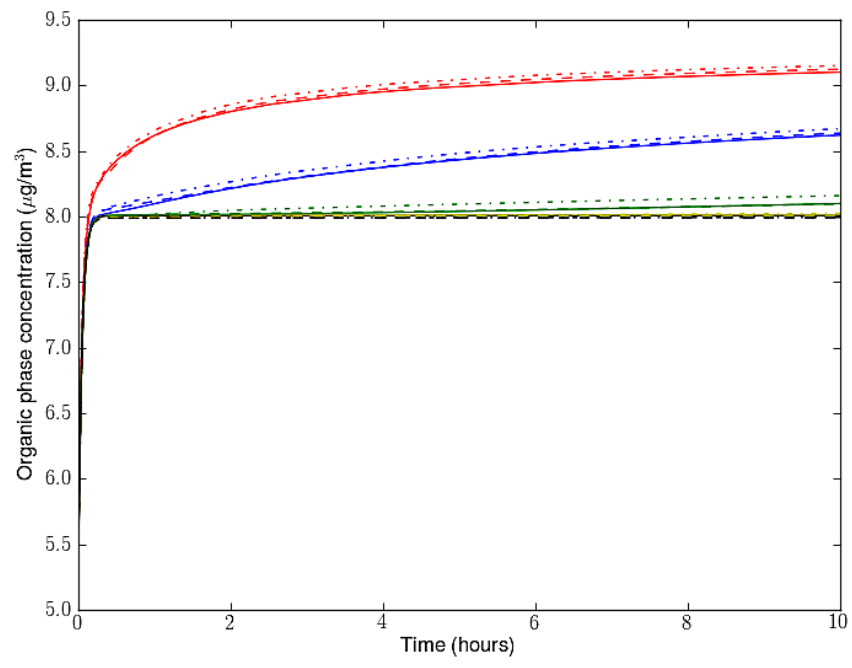

Figure 7. Comparison between the explicit representation (plain lines) and the implicit representation for $N_{\text {layer }}=5$ (- line) and $N_{\text {layer }}=4$ (-. line) for several organic-phase diffusivity: $D_{\text {org }}=10^{-19} \mathrm{~m}^{2} \mathrm{~s}^{-1}$ (red), $D_{\text {org }}=10^{-20} \mathrm{~m}^{2} \mathrm{~s}^{-1}$ (blue), $D_{\text {org }}=$ $10^{-21} \mathrm{~m}^{2} \mathrm{~s}^{-1}$ (green), $D_{\text {org }}=10^{-22} \mathrm{~m}^{2} \mathrm{~s}^{-1}$ (yellow) and $D_{\text {org }}=$ $10^{-24} \mathrm{~m}^{2} \mathrm{~s}^{-1}$ (black) for the compounds from biogenic test case (with an initial concentrations inside the particle equal to $10 \%$ of the concentration at equilibrium) condensing on $5 \mu \mathrm{g} \mathrm{m}^{-3}$ of nonvolatile organic compounds at $\mathrm{RH}=30 \%$. Ideality is assumed.

conditions. BiA2D and BiA1D were assumed hydrophilic based on their octanol/water coefficient (Pun et al., 2006), which is probably not representative of atmospheric conditions. BiMGA was assumed hydrophilic based on the results of Couvidat and Seigneur (2011). According to this study, BiMGA condenses mostly on the aqueous phase if the liquid water content of aerosols is high (superior to the concentration of the organic phase), which is not the case in the two test cases. Moreover, if medium-range and long-range interactions are not taken into account, the distribution of the compounds between phases changes significantly. For the biogenic test case, if medium-range and long-range interactions are not taken into account as in Couvidat and Seigneur (2011), the fraction in the organic phase decreases from 75 to $33 \%$ for particulate BiA2D and from 89 to $46 \%$ for particulate BiA1D. For the anthropogenic test case, the fraction in the organic decreases, from 99 to $76 \%$ for particulate BiA2D, from 100 to $85 \%$ for particulate BiA1D and from 59 to $46 \%$ for particulate BiMGA. It is therefore possible for these compounds to be present in both phases depending on conditions.

\section{Saturation and phase separation}

Species having very different properties do not mix well together and phase separation can be computed by Gibbs energy minimization (see Sect. 2.2.4). Table 9 presents the concentrations with or without phase separation for the bio-
Table 5. Conditions of the test cases.

\begin{tabular}{lcc}
\hline Compounds & $\begin{array}{c}\text { Concentration }\left(\mu \mathrm{g} \mathrm{m}^{-3}\right) \\
\text { Biogenic test case } \\
\text { at } T=295 \mathrm{~K}\end{array}$ & $\begin{array}{c}\text { Concentration }\left(\mu \mathrm{g} \mathrm{m}^{-3}\right) \\
\text { Anthropogenic test case } \\
\text { at } T=293 \mathrm{~K}\end{array}$ \\
\hline BiA2D & 0.07 & 0.02 \\
BiA1D & 0.74 & 0.20 \\
BiA0D & 1.02 & 0.58 \\
BiPER & 1.46 & 0.17 \\
BiDER & 0.45 & 0.06 \\
BiMGA & 0.25 & 0.05 \\
AnBIP & 0.03 & 0.05 \\
AnBmP & 0.27 & 0.32 \\
BiBlP & 0.09 & 0.03 \\
BiBmP & 0.22 & 0.07 \\
AnClP & 0.07 & 0.03 \\
BiNGA & 0.21 & 0.05 \\
BiNIT3 & 0.13 & 0.02 \\
BiNIT & 0.44 & 0.13 \\
POAlP $_{\text {POAmP }}$ & 0.10 & 0.71 \\
POAhP $_{\text {SOAlP }}$ & 0.02 & 0.69 \\
SOAmP & 0.03 & 0.91 \\
SOAhP $_{\mathrm{H}_{2} S \mathrm{SO}_{4}}$ & 0.36 & 0.65 \\
$\mathrm{HNO}_{3}$ & 0.70 & 1.35 \\
$\mathrm{NH}_{3}$ & 1.72 & 2.51 \\
& 1.70 & 1.44 \\
& 1.83 & 5.07 \\
& 1.07 & 1.77 \\
\hline & &
\end{tabular}

genic test case at $\mathrm{RH}=30 \%$ without an aqueous phase. In this case, without phase separation, both hydrophilic and hydrophobic compounds want to condense into the organic phase which is mainly constituted by hydrophobic compounds. Assuming phase separation in this case does not strongly influence concentrations of hydrophobic compounds, which decrease slightly. However, a second organic phase is created by phase separation which is composed of mainly very oxidized compounds (BiPER, BiDER and BiMGA). For the anthropogenic case, at $\mathrm{RH}=30 \%$, phase separation does not occur because the concentrations of hydrophilic compounds are too low for the organic phase to be saturated.

Another organic phase may be created if there are compounds with a low oxidation state. For the biogenic case at low humidity, if the structure of nonadecane is used to represent the compounds POAIP, POAmP, POAhP, SOAIP, SOAmP and SOAhP (without a molecular structure; these compounds only condense into the less oxidized phase without impacting phase separation), a third organic phase may be created. Compounds with a low oxidation state may not readily mix with slightly oxidized compounds which in turn may not readily mix with more oxidized compounds.

\subsection{Dynamic representation}

For dynamic modeling, users must choose the organicphase diffusion coefficients because there is currently, to our knowledge, no method to estimate diffusion coefficients of the organic phase as a function of the composition of organic 
Table 6. Concentrations of organic aerosols formed for each precursor for both test cases at RH=70\% if ideality is assumed.

\begin{tabular}{lcccc}
\hline & \multicolumn{2}{c}{ Biogenic test case } & \multicolumn{2}{c}{ Anthropogenic test cases } \\
& $A_{\mathrm{p}}\left(\mu \mathrm{g} \mathrm{m}^{-3}\right)$ & $A_{\mathrm{aq}}\left(\mu \mathrm{g} \mathrm{m}^{-3}\right)$ & $A_{\mathrm{p}}\left(\mu \mathrm{g} \mathrm{m}^{-3}\right)$ & $A_{\mathrm{aq}}\left(\mu \mathrm{g} \mathrm{m}^{-3}\right)$ \\
\hline Monoterpenes & 0.83 & 1.01 & 0.22 & 0.58 \\
Sesquiterpenes & 0.28 & 0.0 & 0.10 & 0.0 \\
Isoprene & 0.89 & 0.70 & 0.13 & 0.12 \\
Aromatics & 0.09 & 0.05 & 0.09 & 0.04 \\
Primary SVOC & 0.09 & 0 & 0.70 & 0.0 \\
Oxidized primary SVOC & 1.22 & 0 & 2.28 & 0.0 \\
Water & 0.66 & 1.86 & 0.48 & 2.68 \\
\hline
\end{tabular}

Table 7. Concentrations of organic aerosols formed for each precursor for both test cases at $\mathrm{RH}=70 \%$ if non-ideality is assumed (with short-range, medium-range and long-range interactions).

\begin{tabular}{lcccc}
\hline & \multicolumn{2}{c}{ Biogenic test case } & \multicolumn{2}{c}{ Anthropogenic test cases } \\
& $A_{\mathrm{p}}\left(\mu \mathrm{g} \mathrm{m}^{-3}\right)$ & $A_{\mathrm{aq}}\left(\mu \mathrm{g} \mathrm{m}^{-3}\right)$ & $A_{\mathrm{p}}\left(\mu \mathrm{g} \mathrm{m}^{-3}\right)$ & $A_{\mathrm{aq}}\left(\mu \mathrm{g} \mathrm{m}^{-3}\right)$ \\
\hline Monoterpenes & 0.44 & 1.10 & 0.14 & 0.55 \\
Sesquiterpenes & 0.19 & 0.0 & 0.08 & 0.0 \\
Isoprene & 0.18 & 1.24 & 0.02 & 0.13 \\
Aromatics & 0.09 & 0.16 & 0.07 & 0.01 \\
Primary SVOC & 0.08 & 0 & 0.68 & 0.0 \\
Oxidized primary SVOC & 1.04 & 0 & 2.18 & 0.0 \\
Water & 0.06 & 1.05 & 0.06 & 2.75 \\
\hline
\end{tabular}

Table 8. Distributions of surrogate organic compounds between the aqueous phase and the organic phase.

\begin{tabular}{|c|c|c|}
\hline \multirow[t]{2}{*}{ Compound } & \multicolumn{2}{|c|}{ Fraction of the compound in the organic phase (in \%) } \\
\hline & Biogenic test case & Anthropogenic test case \\
\hline BiA2D & 75 & 99 \\
\hline BiA1D & 89 & 100 \\
\hline BiA0D & 0 & 0 \\
\hline BiPER & 2 & 3 \\
\hline BiDER & 2 & 1 \\
\hline BiMGA & 80 & 59 \\
\hline AnBlP & 75 & 100 \\
\hline $\mathrm{AnBmP}$ & 6 & 50 \\
\hline BiBlP & 99 & 100 \\
\hline $\mathrm{BiBmP}$ & 100 & 100 \\
\hline AnClP & 100 & 100 \\
\hline BiNGA & 98 & 99 \\
\hline BiNIT3 & 70 & 97 \\
\hline BiNIT & 97 & 100 \\
\hline
\end{tabular}

aerosols. In this section, the same organic-phase diffusion coefficient is used for all organic compounds. For all the compounds, the gas-phase diffusion coefficient is assumed to be equal to $0.1 \mathrm{~cm}^{2} \mathrm{~s}^{-1}$ which is the order of magnitude of this parameter (Seinfeld and Pandis, 1998) and the accommodation coefficient (value between 0 and 1) is assumed to be equal to 0.01 so that it corresponds to the order of magnitude of accommodation coefficients for organic compounds given in other studies, i.e., between 0.1 (Saleh et al., 2013) and 0.001 (Lee et al., 2011). The gas-phase diffusion coeffi- cient and the accommodation coefficient are used in Eqs. (79) and (80).

In the regional-scale simulations from which initial concentrations are extracted, equilibrium is assumed between gas and particles. For the dynamic modeling, $5 \mu \mathrm{g} \mathrm{m}^{-3}$ of non-volatile hydrophobic organic compounds is added to have a mass into which compounds are not present and will have to diffuse. For each surrogate, the total mass gas + particles is kept the same as in the equilibrium simulations. However, the particle-phase concentrations are assumed to be equal to $10 \%$ of the equilibrium particle-phase concentrations.

To fully validate the implicit representation of the dynamic evolution of surrogates, the results of SOAP are compared to the results of the explicit representation for the biogenic test case assuming ideality. $\mathrm{RH}$ is assumed to be equal to $30 \%$. Results of this comparison are shown in Fig. 7. This results shows good results with five layers and a slight overestimation with four layers of OM concentrations due to the overestimation of condensation for low volatility compounds.

The dynamic implicit representation is tested for various humidities and various diffusion coefficients for the biogenic and anthropogenic cases. Figures 8 and 9 show the concentrations in the organic phases for the biogenic and anthropogenic cases, respectively, whereas Figs. 10 and 11 show concentrations in the aqueous phase for the biogenic and anthropogenic cases, respectively. The influence of the number of layers is also tested in these figures. 
Table 9. Concentrations (in $\mu \mathrm{g} \mathrm{m}^{-3}$ ) with or without phase separation for the biogenic test case at $\mathrm{RH}=30 \%$.

\begin{tabular}{lcccc}
\hline Compound & Without phase separation & \multicolumn{3}{c}{ With phase separation } \\
& $A_{\mathrm{p}}$ & 1st phase & 2nd phase & All phases \\
\hline BiA2D & 0.042 & 0.037 & 0.005 & 0.042 \\
BiA1D & 0.322 & 0.299 & 0.021 & 0.320 \\
BiA0D & 0.001 & 0.0 & 0.001 & 0.0 \\
BiPER & 0.031 & 0.026 & 0.030 & 0.056 \\
BiDER & 0.015 & 0.011 & 0.048 & 0.059 \\
BiMGA & 0.027 & 0.020 & 0.040 & 0.060 \\
AnB1P & 0.012 & 0.011 & 0.001 & 0.012 \\
AnBmP & 0.012 & 0.010 & 0.010 & 0.020 \\
BiBlP & 0.092 & 0.091 & 0.001 & 0.092 \\
BiBmP & 0.098 & 0.096 & 0.0 & 0.096 \\
AnClP & 0.069 & 0.069 & 0.0 & 0.069 \\
BiNGA & 0.062 & 0.051 & 0.020 & 0.071 \\
BiNIT3 & 0.010 & 0.010 & 0.001 & 0.011 \\
BiNIT & 0.043 & 0.041 & 0.001 & 0.042 \\
POAlP & 0.076 & 0.075 & 0.0 & 0.075 \\
POAmP & 0.001 & 0.0 & 0.0 & 0.0 \\
POAhP & 0.0 & 0.0 & 0.0 & 0.0 \\
SOAlP & 0.356 & 0.356 & 0.0 & 0.356 \\
SOAmP & 0.532 & 0.527 & 0.0 & 0.527 \\
SOAhP & 0.132 & 0.127 & 0.0 & 0.127 \\
Water & 0.020 & 0.018 & 0.012 & 0.030 \\
\hline Sum of organics & 1.933 & 1.857 & 0.173 & 2.035 \\
\hline & & & &
\end{tabular}

The two test cases show similar results. For the condensation into the organic phases, the growth of particles can be divided into two parts. The particle grows by condensation into the layer at the interface independently from the viscosity of the organic phases until the growth of particle become limited by the diffusion of the more volatile compounds into the organic phases. For the anthropogenic test case, humidity has a low effect between 30 and $70 \%$ on the condensation of organic compounds into the organic phases because they are mainly constituted of hydrophobic compounds in the anthropogenic test case. However, at $70 \%$ more hydrophilic compounds manage to condense into the aqueous phase. Between 70 to $90 \%$, the amount of organic compounds in the organic phases decreases whereas the amount of organic compounds increases in the aqueous phase. For the biogenic test case, the organic-phase concentration increases between 30 and $50 \%$ of humidity because hydrophilic compounds (which are in higher concentrations than in the anthropogenic test case) partition more efficiently. Between 30 and $70 \%$, the organicphase concentration decreases but the aqueous-phase concentration increases because hydrophilic compounds condense more efficiently into the aqueous phase. However, diffusion coefficients are probably much higher at high humidity than at low humidity because the organic phases would be less viscous (less oligomerization due to esterification for example and more water in the organic phases which would decrease viscosity) as shown by Saukko et al. (2012) and
Renbaum-Wolff et al. (2013). It may then be possible that at high humidity, organic aerosols reach equilibrium quicker than at low humidity. A method to estimate diffusion coefficients as a function of composition (or at least of as a function of humidity) is needed to properly represent this phenomenon.

\subsection{Time analysis}

Table 10 shows the computation time necessary to solve the biogenic case at $\mathrm{RH}=70 \%$ (relative to the time necessary to solve the case with an uncoupled and ideal system) for different configurations of the model. For the dynamic approach, 4 layers are used, the length of the computation corresponds to $600 \mathrm{~s}$ (corresponding to a time step of a simulation with the Polyphemus air quality platform over Europe; Sartelet et al., 2007), initial concentrations are assumed to be $80 \%$ of concentrations at equilibrium and an organic-phase diffusion coefficient of $10^{-21} \mathrm{~m}^{2} \mathrm{~s}^{-1}$ is used. The times given here correspond to a specific case and therefore can greatly vary with initial conditions and with the chosen parameters for the numerical resolution of the system. They are provided here on an indicative basis. The dynamic approach is around 400 times slower than the equilibrium approach, making its applicability limited to short-term 3-D simulations. 
$\mathrm{RH}=30 \%$

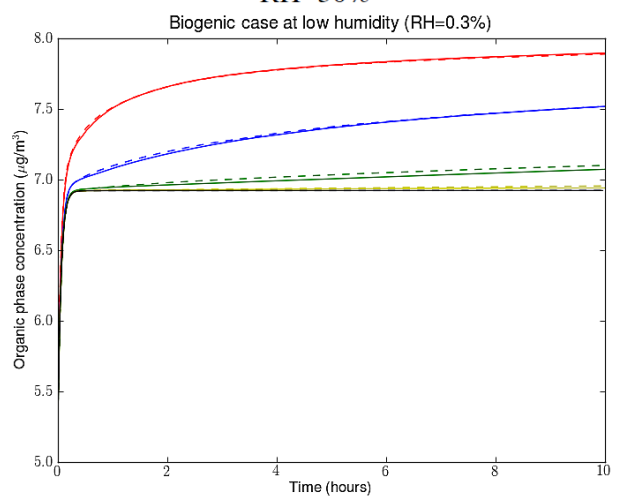

$\mathrm{RH}=70 \%$

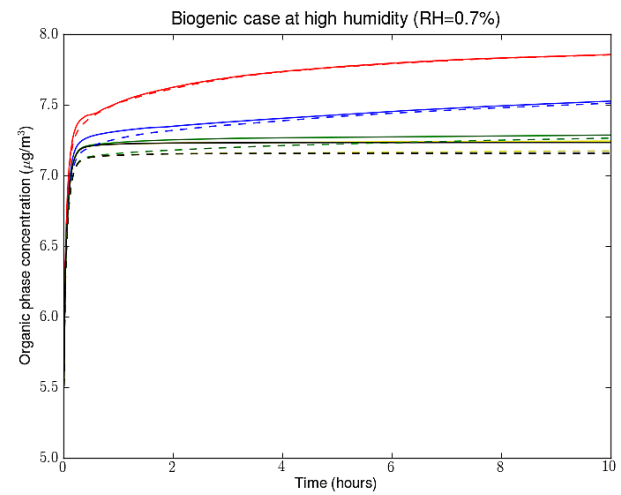

$\mathrm{RH}=50 \%$

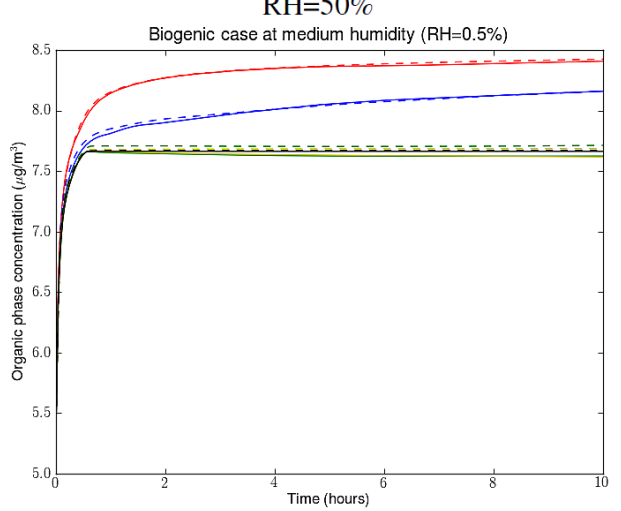

$\mathrm{RH}=90 \%$

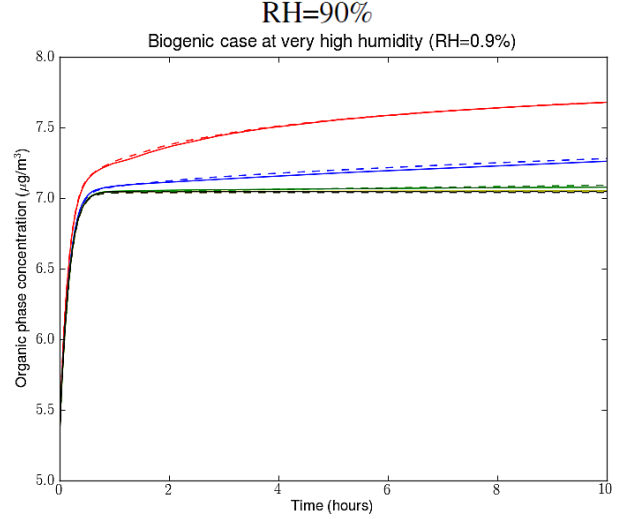

Figure 8. Evolution of concentrations in organic phases for the biogenic case at various humidities, with initial concentrations equal to one tenth of the concentrations at equilibrium and $5 \mu \mathrm{g} \mathrm{m}^{-3}$ of non-volatile compounds for $N_{\text {layer }}=5$ (plain lines) and $N_{\text {layer }}=4$ (dashed lines) for several organic-phase diffusivity: $D_{\mathrm{org}}=10^{-19} \mathrm{~m}^{2} \mathrm{~s}^{-1}$ (red), $D_{\text {org }}=10^{-20} \mathrm{~m}^{2} \mathrm{~s}^{-1}$ (blue), $D_{\mathrm{org}}=10^{-21} \mathrm{~m}^{2} \mathrm{~s}{ }^{-1}$ (green), $D_{\text {org }}=$ $10^{-22} \mathrm{~m}^{2} \mathrm{~s}^{-1}$ (yellow) and $D_{\text {org }}=10^{-24} \mathrm{~m}^{2} \mathrm{~s}^{-1}$ (black).

Table 10. Time necessary to solve the biogenic case at $\mathrm{RH}=70 \%$ (relative to the time necessary to solve the case with an uncoupled system with ideality) for different configurations of the model.

\begin{tabular}{|c|c|c|c|c|c|}
\hline \multicolumn{4}{|c|}{ Phenomena taken into account } & \multicolumn{2}{|c|}{ Time for } \\
\hline $\begin{array}{l}\text { Coupled } \\
\text { system }\end{array}$ & $\begin{array}{l}\text { Short-range } \\
\text { interactions }\end{array}$ & $\begin{array}{l}\text { Long- and medium- } \\
\text { range interactions }\end{array}$ & $\begin{array}{l}\text { Organic phase } \\
\text { separation }\end{array}$ & $\begin{array}{r}\text { Equilibrium } \\
\text { approach }\end{array}$ & $\begin{array}{l}\text { Dynamic } \\
\text { approach }\end{array}$ \\
\hline & & & & 1 & 400 \\
\hline $\mathrm{X}$ & & & & 1.4 & 800 \\
\hline & $\mathrm{X}$ & & & 4.2 & 2400 \\
\hline $\mathrm{X}$ & $X$ & & & 18 & 4400 \\
\hline & $X$ & $X$ & & 7 & 2500 \\
\hline $\mathrm{X}$ & $\mathrm{X}$ & $X$ & & 23 & 5400 \\
\hline & $\mathrm{X}$ & $\mathrm{X}$ & $\mathrm{X}$ & 23 & 2600 \\
\hline $\mathrm{X}$ & $\mathrm{X}$ & $\mathrm{X}$ & $\mathrm{X}$ & 79 & 66000 \\
\hline
\end{tabular}

More reliable and complete information about the computation time cost will be provided in future studies about the implementation of the SOAP in an air quality model.

\section{Conclusions}

The Secondary Organic Aerosol Processor (SOAP) model is a modular model, which can compute the condensationevaporation of organic aerosol according to two different approaches: an equilibrium approach and a dynamic ap- 

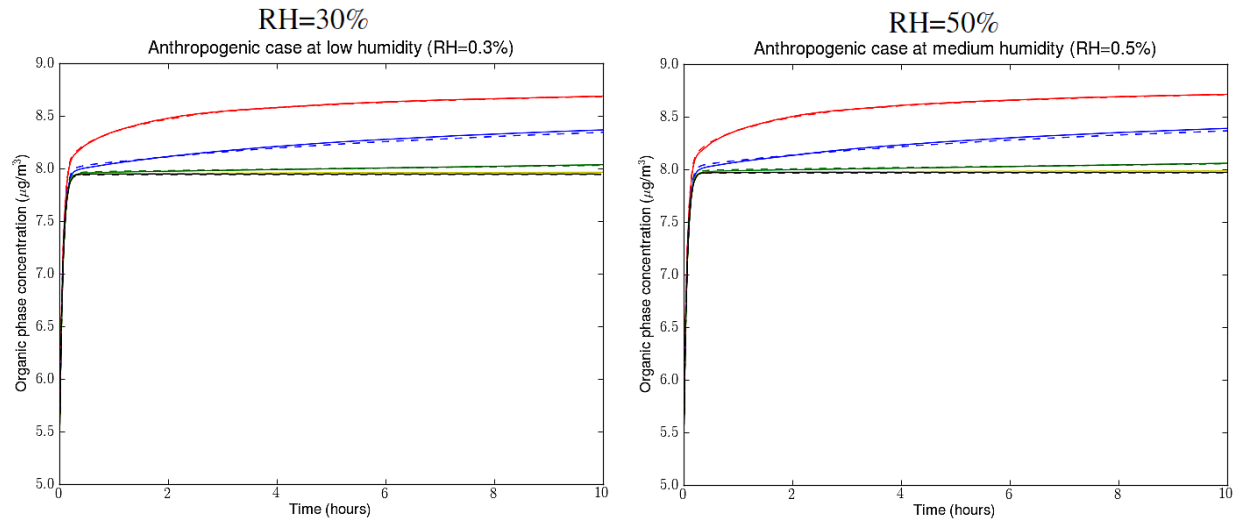

$\mathrm{RH}=70 \%$
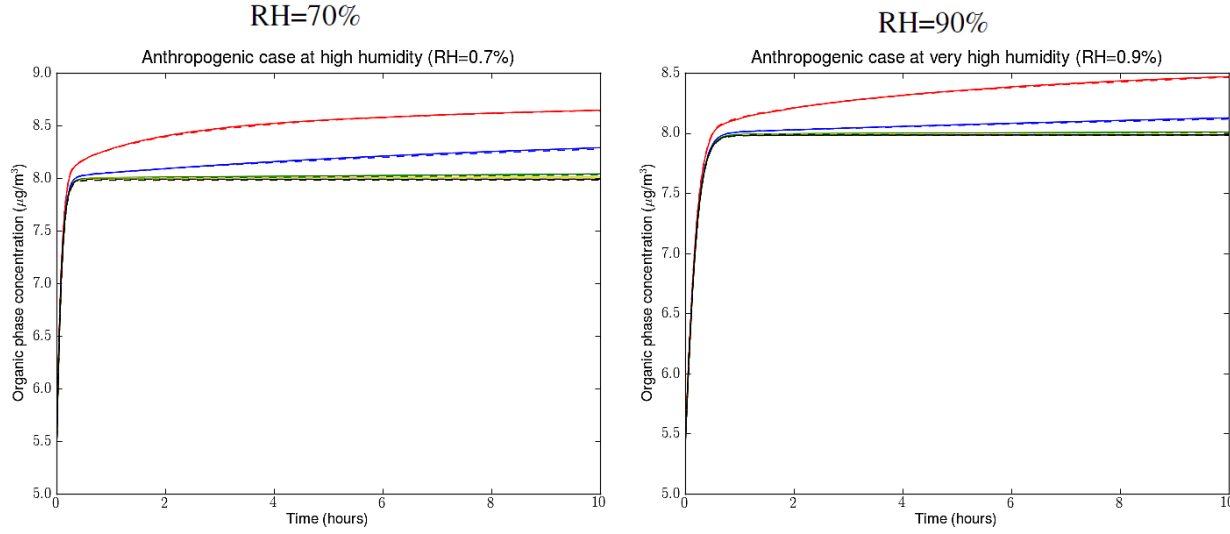

Figure 9. Evolution of concentrations in organic phases for the anthropogenic case at various humidities, with initial concentrations equal to one tenth of the concentrations at equilibrium and $5 \mu \mathrm{g} \mathrm{m}^{-3}$ of non-volatile compounds for $N_{\text {layer }}=5$ (plain lines) and $N_{\text {layer }}=4$ (dashed lines) for several organic-phase diffusivity: $D_{\text {org }}=10^{-19} \mathrm{~m}^{2} \mathrm{~s}^{-1}$ (red), $D_{\text {org }}=10^{-20} \mathrm{~m}^{2} \mathrm{~s}^{-1}$ (blue), $D_{\mathrm{org}}=10^{-21} \mathrm{~m}^{2} \mathrm{~s}^{-1}(\mathrm{green}), D_{\mathrm{org}}=$ $10^{-22} \mathrm{~m}^{2} \mathrm{~s}^{-1}$ (yellow) and $D_{\text {org }}=10^{-24} \mathrm{~m}^{2} \mathrm{~s}^{-1}$ (black).

$\mathrm{RH}=70 \%$

Biogenic case at high humidity $(\mathrm{RH}=0.7 \%)$

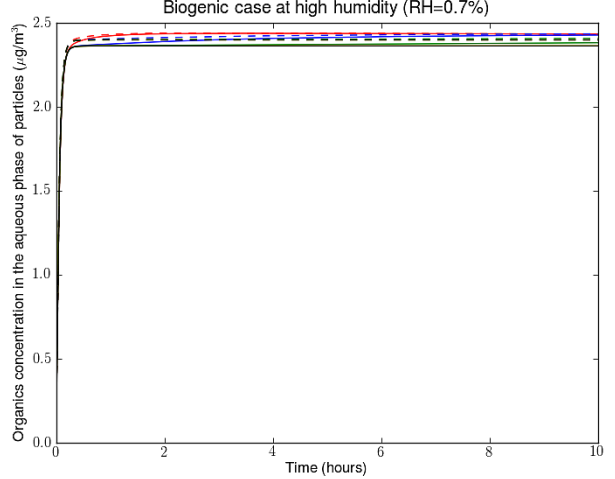

$\mathrm{RH}=90 \%$

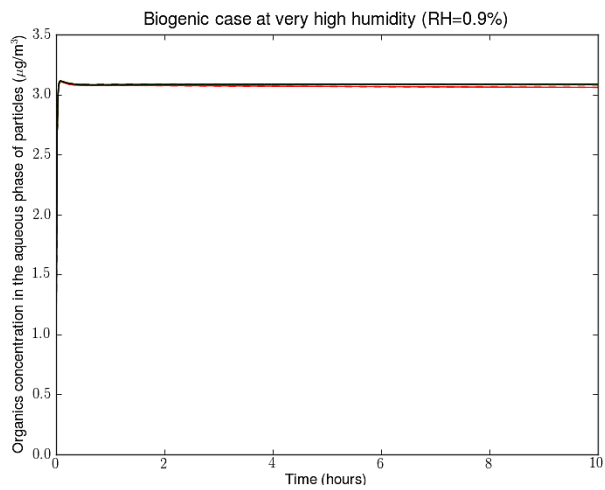

Figure 10. Evolution of concentrations in the aqueous phase for the biogenic case at various humidities, with initial concentrations equal to one tenth of the concentrations at equilibrium and $5 \mu \mathrm{g} \mathrm{m}^{-3}$ of non-volatile compounds for $N_{\text {layer }}=5$ (plain lines) and $N_{\text {layer }}=4$ (dashed lines) for several organic-phase diffusivity: $D_{\text {org }}=10^{-19} \mathrm{~m}^{2} \mathrm{~s}^{-1}$ (red), $D_{\text {org }}=10^{-20} \mathrm{~m}^{2} \mathrm{~s}^{-1}$ (blue), $D_{\text {org }}=10^{-21} \mathrm{~m}^{2} \mathrm{~s}^{-1}(\mathrm{green}), D_{\mathrm{org}}=$ $10^{-22} \mathrm{~m}^{2} \mathrm{~s}^{-1}$ (yellow) and $D_{\text {org }}=10^{-24} \mathrm{~m}^{2} \mathrm{~s}^{-1}$ (black). 

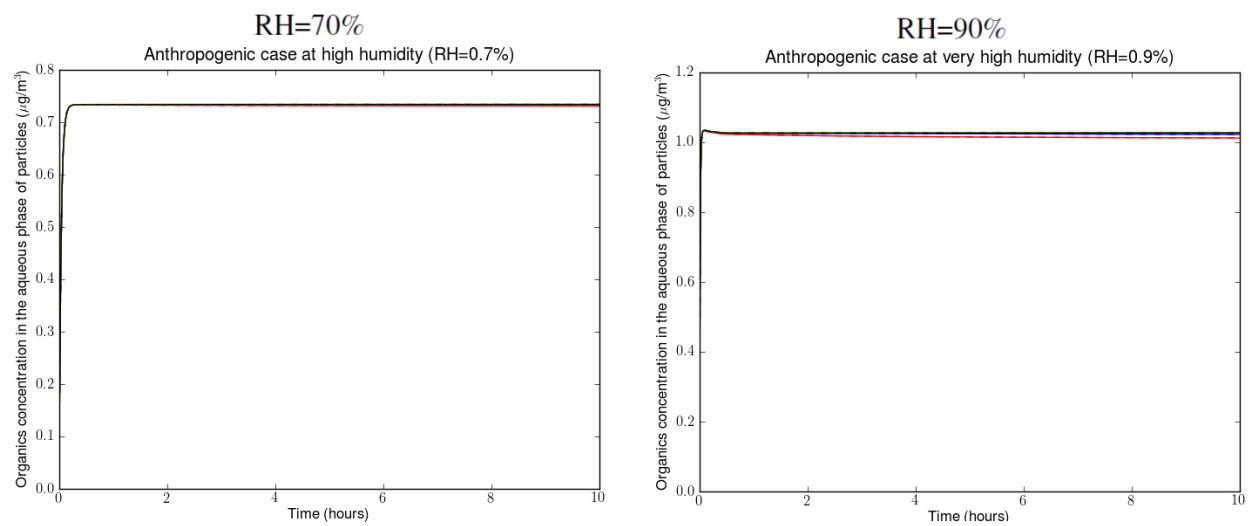

Figure 11. Evolution of concentrations in the aqueous phase for the anthropogenic case at various humidities, with initial concentrations equal to one tenth of the concentrations at equilibrium and $5 \mu \mathrm{g} \mathrm{m}^{-3}$ of non-volatile compounds for $N_{\text {layer }}=5$ (plain lines) and $N_{\text {layer }}=4$ (dashed lines) for several organic-phase diffusivity: $D_{\mathrm{org}}=10^{-19} \mathrm{~m}^{2} \mathrm{~s}^{-1}$ (red), $D_{\mathrm{org}}=10^{-20} \mathrm{~m}^{2} \mathrm{~s}^{-1}$ (blue), $D_{\mathrm{org}}=10^{-21} \mathrm{~m}^{2} \mathrm{~s}-1$ (green), $D_{\text {org }}=10^{-22} \mathrm{~m}^{2} \mathrm{~s}^{-1}$ (yellow) and $D_{\text {org }}=10^{-24} \mathrm{~m}^{2} \mathrm{~s}^{-1}$ (black).

proach. In the equilibrium approach, concentrations in the particle phase are assumed at equilibrium with concentrations in the gas phase. In the dynamic approach, concentrations evolve according to the kinetics of condensationevaporation-diffusion of organic compounds. The dynamic approach uses a multi-layer representation of particles to represent the particle-phase diffusion of organic compounds. Future works will focus on improving the framework of this dynamic approach to take into account varying diffusion coefficients with layers, to allow for the entrapment of compounds in inner layers and to represent layer exchanges and transfers between the organic and the aqueous phases. Simulations with SOAP and comparisons to measurements should be performed to validate the model and to test the influence of each process and parameter on organic aerosol formation.
To improve the representation of aerosols, several processes should be added to the model. First, interactions between organic and inorganic compounds should be fully taken into account via activity coefficients. Currently, only the influence of inorganic compounds on organic compounds is taken into account. However, organic compounds can also impact the formation of inorganic compounds due to those interactions. This process could be taken into account by adding inorganic aerosol formation to SOAP. Second, a method to estimate diffusion coefficients in organic phases should be developed, as it is expected that composition of the organic phases greatly influences the viscosity and therefore diffusion coefficients of organic compounds. Finally, the model could be coupled to a solver for particle-phase chemistry and then represent processes such as oligomerization, which could affect the viscosity of the organic phases. 


\section{Appendix A: Notations}

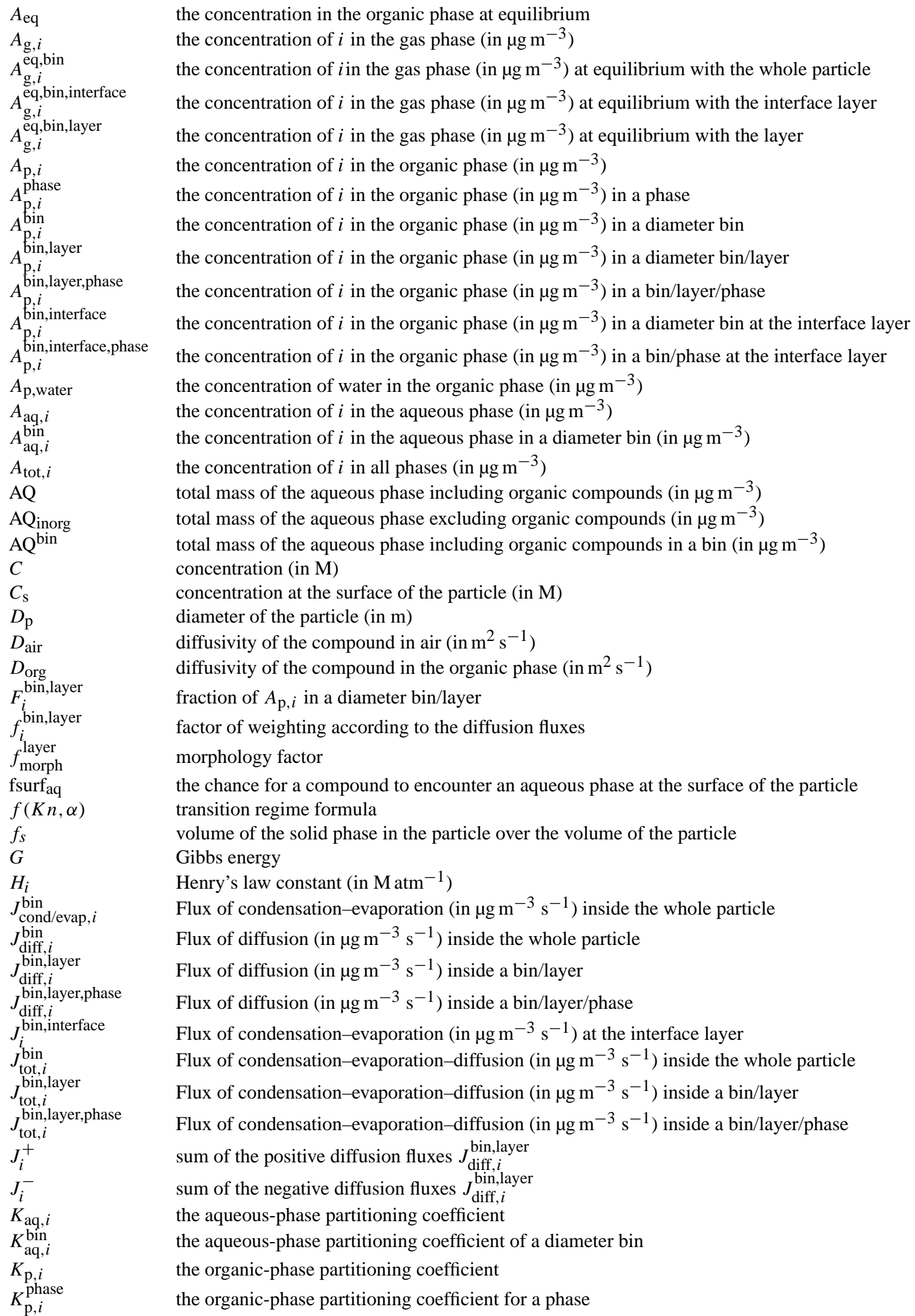




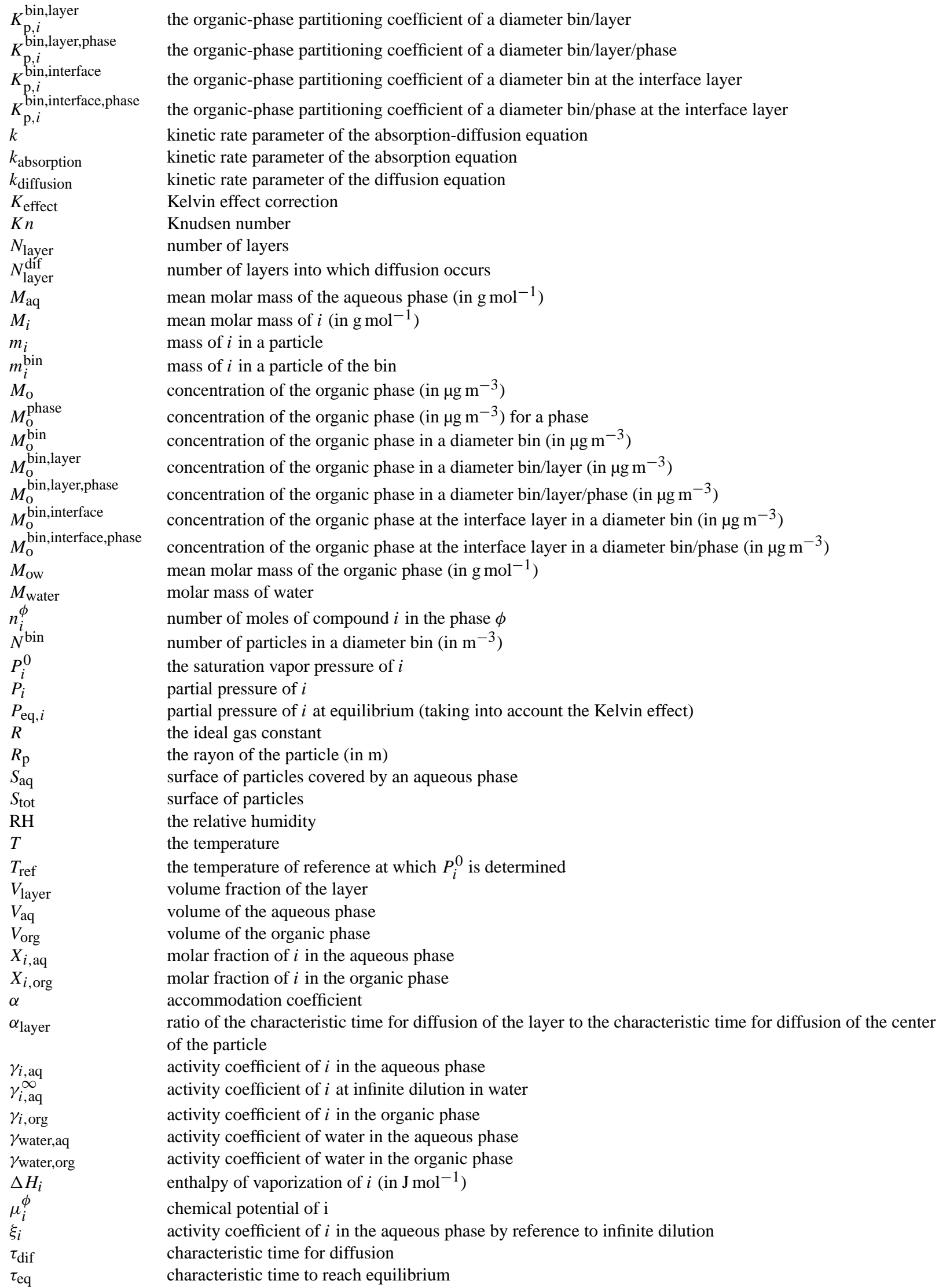




\section{The Supplement related to this article is available online at doi:10.5194/gmd-8-1111-2015-supplement.}

Acknowledgements. This work was funded by the french ministry of ecology, by CNRS-INSU (GMES-MDD program) and by ADEME, the French Agency for the Environment and Energy Management.

Edited by: H. Tost

\section{References}

Abramson, E., Imre, D., Beránek, J., Wilson, J., and Zelenyuk, A.: Experimental determination of chemical diffusion with secondary organic aerosol particles, Phys. Chem. Chem. Phys., 15, 2983-2991, doi:10.1039/C2CP44013J, 2013.

Capaldo, K. P., Pilinis, C., and Pandis, S. N.: A computationally efficient hydbrid approach for dynamic gas/aerosol transfer in air quality models, Atmos. Environ., 34, 3617-3627, doi:10.1016/S1352-2310(00)00092-3, 2000.

Cappa, C. D. and Wilson, K. R.: Evolution of organic aerosol mass spectra upon heating: implications for OA phase and partitioning behavior, Atmos. Chem. Phys., 11, 1895-1911, doi:10.5194/acp11-1895-2011, 2011.

Choi, M. Y. and Chan, C. K.: The effects of organic species on the hygroscopic behaviors of inorganic aerosols, Environ. Sci. Technol., 36, 2422-2428, doi:10.1021/es0113293, 2002.

Couvidat, F. and Seigneur, C.: Modeling secondary organic aerosol formation from isoprene oxidation under dry and humid conditions, Atmos. Chem. Phys., 11, 893-909, doi:10.5194/acp-11893-2011, 2011.

Couvidat, F., Debry, É., Sartelet, K., and Seigneur, C.: A Hydrophilic/Hydrophobic Organic $\left(\mathrm{H}^{2} \mathrm{O}\right)$ model: Model development, evaluation and sensitivity analysis, J. Geophys. Res., 117, D10 304, doi:10.1029/2011JD017214, 2012.

Couvidat, F., Kim, Y., Sartelet, K., Seigneur, C., Marchand, N., and Sciare, J.: Modeling secondary organic aerosol in an urban area: application to Paris, France, Atmos. Chem. Phys., 13, 983-996, doi:10.5194/acp-13-983-2013, 2013.

EPRI: Organic Aerosol Partition Module Documentation, EPRI, Palo Alto, CA, USA, Technical Report, 1999.

Erdakos, G. B. and Pankow, J. F.: Gas/particle partitioning of neutral and ionizing compounds to single and multi-phase aerosol particles. 2. Phase separation in liquid particulate matter containing both polar and low-polarity organic compounds, Atmos. Environ., 38, 1005-1013, doi:10.1016/j.atmosenv.2003.10.038, 2004.

Fredenslund, A., Jones, R. L., and Prausnitz, J. M.: Groupcontribution estimation of activity-coefficients in nonideal liquid-mixtures, AIChE J., 21, 1086-1099, 1975.

Fuchs, N. and Sutugin, A.: Topics in current aerosol research (part 2), Chapt. High dispersed aerosols, Pergamon, New York, 1971.

Griffin, R. J., Nguyen, K., Dabdub, D., and Seinfeld, J. H.: A coupled hydrophobic-hydrophilic model for predicting secondary organic aerosol formation, J. Atmos. Chem., 44, 171190, doi:10.1023/A:1022436813699, 2003.
Kanakidou, M., Seinfeld, J. H., Pandis, S. N., Barnes, I., Dentener, F. J., Facchini, M. C., Van Dingenen, R., Ervens, B., Nenes, A., Nielsen, C. J., Swietlicki, E., Putaud, J. P., Balkanski, Y., Fuzzi, S., Horth, J., Moortgat, G. K., Winterhalter, R., Myhre, C. E. L., Tsigaridis, K., Vignati, E., Stephanou, E. G., and Wilson, J.: Organic aerosol and global climate modelling: a review, Atmos. Chem. Phys., 5, 1053-1123, doi:10.5194/acp-5-1053-2005, 2005.

Katrinak, K. A., Rez, P., Perkes, P. R., and Buseck, P. R.: Fractal geometry of carbonaceous agregates from an urban aerosol, Environ. Sci. Technol., 27, 539-547, doi:10.1021/es00040a013, 1993.

Larson, S. M., Cass, G. R., and Gray, A. H.: Atmospheric Carbon Particles and the Los Angeles Visibility Problem, Aerosol Sci. Technol., 10, 118-130, doi:10.1080/02786828908959227, 1989.

Lee, B.-H., Pierce, J. R., Engelhart, G. J., and Pandis, S. N.: Volatility of secondary organic aerosol from the ozonolysis of monoterpenes, Atmos. Environ., 45, 2443-2452, doi:10.1016/j.atmosenv.2011.02.004, 2011

Nenes, A., Pandis, S. N., and Pilinis, C.: ISORROPIA: A new thermodynamic equilibrium model for multiphase multicomponent inorganic aerosols, Aquat. Geoch., 4, 123-152, 1998.

Pankow, J. F.: An absorption model of gas/particle partitioning of organic compounds in the atmosphere, Atmos. Environ., 28A, 185-188, 1994.

Pfrang, C., Shiraiwa, M., and Pöschl, U.: Chemical ageing and transformation of diffusivity in semi-solid multi-component organic aerosol particles, Atmos. Chem. Phys., 11, 7343-7354, doi:10.5194/acp-11-7343-2011, 2011.

Pun, B. K., Griffin, R. J., Seigneur, C., and Seinfeld, J. H.: Secondary organic aerosol. 2. Thermodynamic model for gas/particle partitioning of molecular constituents, J. Geophys. Res., 107, 433, doi:10.1029/2001JD000542, 2002.

Pun, B. K., Seigneur, C., and Lohman, K.: Modeling secondary organic aerosol formation via multiphase partitioning with molecular data, Environ. Sci. Technol., 40, 4722-4731, doi:10.1021/es0522736, 2006.

Renbaum-Wolff, L., Grayson, J. W., Bateman, A. P., Kuwata, M., Sellier, M., Murray, B. J., Shilling, J. E., Martin, S. T., and Bertram, A. K.: Viscosity of $\alpha$-pinene secondary organic material and implications for particle growth and reactivity, Proc. Natl. Acad. Sci., 110, 8014-8019, doi:10.1073/pnas.1219548110, 2013.

Robinson, R. A. and Stokes, R. H.: Electrolyte solutions, 2nd (revised) Edn., Butterworth, 1965.

Sachdeva, K. and Attri, A. K.: Morphological characterization of carbonaceous aggregates in soot and free fall aerosol samples, Atmos. Environ., 42, 1025-1034, doi:10.1016/j.atmosenv.2007.10.002, 2007.

Saleh, E., Donahue, N. M., and Robinson, A. L.: Time Scales for Gas-Particle Partitioning Equilibration of Secondary Organic Aerosol Formed from Alpha-Pinene Ozonolysis, Environ. Sci. Technol., 47, 5588-5594, doi:10.1021/es400078d, 2013.

Sartelet, K., Debry, E., Fahey, K., Roustan, Y., Tombette, M., and Sportisse, B.: Simulation of aerosols and gas-phase species over Europe with the Polyphemus system, Part I: model-todata comparison for 2001, Atmos. Environ., 41, 6116-6131, doi:10.1016/j.atmosenv.2007.04.024, 2007. 
Saukko, E., Lambe, A. T., Massoli, P., Koop, T., Wright, J. P., Croasdale, D. R., Pedernera, D. A., Onasch, T. B., Laaksonen, A., Davidovits, P., Worsnop, D. R., and Virtanen, A.: Humiditydependent phase state of SOA particles from biogenic and anthropogenic precursors, Atmos. Chem. Phys., 12, 7517-7529, doi:10.5194/acp-12-7517-2012, 2012.

Seinfeld, J. and Pandis, S.: Atmospheric chemistry and physics: from air pollution to climate change, Wiley-interscience, 1998.

Shiraiwa, M. and Seinfeld, J. H.: Equilibration timescale of atmospheric secondary organic aerosol partitioning, Geophys. Res. Lett., 39, L24801, doi:10.1029/2012GL054008, 2012.

Shiraiwa, M., Ammann, M., Koop, T., and Pöschl, U.: Gas uptake and chemical aging of semisolid organic aerosol particles, Proc. Natl. Acad. Sci., 108, 11003-11008, doi:10.1073/pnas.1103045108, 2011.

Shiraiwa, M., Pfrang, C., Koop, T., and Pöschl, U.: Kinetic multilayer model of gas-particle interactions in aerosols and clouds (KM-GAP): linking condensation, evaporation and chemical reactions of organics, oxidants and water, Atmos. Chem. Phys., 12, 2777-2794, doi:10.5194/acp-12-2777-2012, 2012.

Shiraiwa, M., Zuend, A., Bertram, A. K., and Seinfeld, J. H.: Gasparticle partitioning of atmospheric aerosols: interplay of physical mixing and morphology, Phys. Chem. Chem. Phys., 15, 11441-11453, doi:10.1039/c3cp51595h, 2013.

Vaden, T. D., Imre, D., Berankek, J., Shrivastava, M., and Zelenyuk, A.: Evaporation kinetics and phase of laboratory and ambient secondary organic aerosol, Proc. Natl. Acad. Sci., 108, 21902195, doi:10.1073/pnas.1013391108, 2011.

Verwer, J. G., Spee, E. J., Bloom, J. G., and Hundsdorfer, W.: A second-order rosenbrock method applied to photochemical dispersion problems, SIAM J. Sci. Comput., 20, 1456-1480, doi:10.1137/S1064827597326651, 1999.

Virtanen, A., Joutsensaari, J., Koop, T., Kannosto, J., YliPirilä, P., Leskinen, J., Mäkelä, J. M., Holopainen, J. K., Pöschl, U., Kulmala, M., Worsnop, D. R., and Laaksonen, A.: An amorphous solid state of biogenic secondary organic aerosol particles, Nature, 467, 824-827, doi:10.1038/nature09455, 2010.

Wang, Y., Sartelet, K. N., Bocquet, M., and Chazette, P.: Modelling and assimilation of lidar signals over Greater Paris during the MEGAPOLI summer campaign, Atmos. Chem. Phys., 14, 35113532, doi:10.5194/acp-14-3511-2014, 2014.
WHO: Health Aspects of Air Pollution with Particulate Matter, Ozone and Nitrogen Dioxide, Tech. rep., WHO EUR/03/5042688, 2003.

Yu, S., Bhave, P. V., Dennis, R. L., and Marthur, R.: Seasonal and regional variations of primary and secondary organic aerosols over the Continental United States: Semi-empirical estimates and model evaluation, Environ. Sci. Technol., 41, 4690-4697, doi:10.1021/es061535g, 2007.

Zhang, Q., Jimenez, J. L., Canagaratna, M. R., Allan, J. D., Coe, H., Ulbrich, I., Alfarra, M. R., Takami, A., Middlebrook, A. M., Sun, Y. L., Dzepina, K., Dunlea, E., Docherty, K., DeCarlo, P. F., Salcedo, D., Onasch, T., Jayne, J. T., Miyoshi, T., Shimono, A., Hatakeyama, S., Takegawa, N., Kondo, Y., Schneider, J., Drewnick, F., Borrmann, S., Weimer, S., Demerjian, K., Williams, P., Bower, K., Bahreini, R., Cottrell, L., Griffin, R. J., Rautiainen, J., Sun, J. Y., Zhang, Y. M., and Worsnop, D. R.: Ubiquity and dominance of oxygenated species in organic aerosols in anthropogenically-influence Northern Hemisphere mildlatitudes, Geophys. Res. Lett., 34, L13801, doi:10.1029/2007GL029979, 2007.

Zuend, A. and Seinfeld, J. H.: Modeling the gas-particle partitioning of secondary organic aerosol: the importance of liquidliquid phase separation, Atmos. Chem. Phys., 12, 3857-3882, doi:10.5194/acp-12-3857-2012, 2012.

Zuend, A., Marcolli, C., Luo, B. P., and Peter, T.: A thermodynamic model of mixed organic-inorganic aerosols to predict activity coefficients, Atmos. Chem. Phys., 8, 4559-4593, doi:10.5194/acp8-4559-2008, 2008.

Zuend, A., Marcolli, C., Peter, T., and Seinfeld, J. H.: Computation of liquid-liquid equilibria and phase stabilities: implications for RH-dependent gas/particle partitioning of organic-inorganic aerosols, Atmos. Chem. Phys., 10, 7795-7820, doi:10.5194/acp10-7795-2010, 2010.

Zuend, A., Marcolli, C., Booth, A. M., Lienhard, D. M., Soonsin, V., Krieger, U. K., Topping, D. O., McFiggans, G., Peter, T., and Seinfeld, J. H.: New and extended parameterization of the thermodynamic model AIOMFAC: calculation of activity coefficients for organic-inorganic mixtures containing carboxyl, hydroxyl, carbonyl, ether, ester, alkenyl, alkyl, and aromatic functional groups, Atmos. Chem. Phys., 11, 9155-9206, doi:10.5194/acp11-9155-2011, 2011. 